\title{
Exposure of the North Atlantic right whale Eubalaena glacialis to the marine algal biotoxin, domoic acid
}

\author{
Luis F. Leandro ${ }^{1,2}$, Rosalind M. Rolland ${ }^{3}$, Patricia B. Roth $^{2}$, Nina Lundholm ${ }^{4}$, \\ Zhihong Wang ${ }^{1}$, Gregory J. Doucette ${ }^{1, *}$ \\ ${ }^{1}$ Marine Biotoxins Program, NOAA/National Ocean Service, 219 Fort Johnson Rd., Charleston, South Carolina 29412, USA \\ ${ }^{2}$ Grice Marine Laboratory, College of Charleston, 205 Fort Johnson Rd., Charleston, South Carolina 29412, USA \\ ${ }^{3}$ Research Department, New England Aquarium, Central Wharf, Boston, Massachusetts 02110, USA \\ ${ }^{4}$ Section of Aquatic Biology, Biological Institute, University of Copenhagen, Øster Farimagsgade 2D, 1353 Copenhagen K, \\ Denmark
}

\begin{abstract}
In addition to ship collisions and fishing gear entanglements, recovery of the highly endangered North Atlantic right whale Eubalaena glacialis has been challenged by reproductive abnormalities and compromised health. Of the factors hypothesized as contributing to the observed reproductive dysfunction in right whales, exposure to marine biotoxins such as domoic acid (DA) has received comparatively little consideration. The present study assessed the occurrence of DA in right whale feces, copepods, and krill collected from April through September of 2005 and 2006 on the whales' feeding grounds along the northeastern USA and eastern Canada. DA was detected by surface plasmon resonance (SPR) in 69 right whale fecal samples, 6 krill samples, and 32 copepod samples. Many of the latter were dominated largely by Stage V Calanus finmarchicus copepodites. DA detection by SPR in fecal and zooplankton collections was verified by receptor binding assay and confirmed using liquid chromatography-tandem mass spectrometry (LC-MS/MS). Moreover, LCMS/MS determined the presence of a putative methylated DA metabolite in all fecal and zooplankton samples analyzed. Frustules of several potentially toxic Pseudo-nitzschia spp. were identified in whale feces and phytoplankton samples by light and electron microscopy. Electron microscopy also revealed an abundance of $C$. finmarchicus mandibles in right whale feces. These findings confirm that E. glacialis was exposed to DA for several months, likely through ingestion of a DAcontaminated copepod vector. The extent to which this algal biotoxin may contribute to the failed recovery of the E. glacialis population warrants further investigation.
\end{abstract}

KEY WORDS: Domoic acid · Eubalaena glacialis $\cdot$ Right whale $\cdot$ Pseudo-nitzschia $\cdot$ Calanus finmarchicus $\cdot$ Copepod $\cdot$ Biotoxin trophic transfer $\cdot$ Harmful algal blooms

\section{INTRODUCTION}

The North Atlantic right whale Eubalaena glacialis is one of the most endangered whale species, with a current population estimated at roughly 350 to 400 individuals (Clapham et al. 1999, Hamilton et al. 2007). Despite efforts to conserve this endangered species, little recovery has occurred since the whaling ban put in place during the 1930s (Knowlton \& Kraus 2001,
Greene \& Pershing 2004, Kraus \& Rolland 2007). Moreover, population growth models have shown that the North Atlantic right whale population was in decline throughout the 1990s and could become extinct in less than 2 centuries if current trends persist (Caswell et al. 1999, Fujiwara \& Caswell 2001). Among the major threats to conserving the E. glacialis population are anthropogenic factors, of which ship collisions and fishing gear entanglements are the main contributors 
(Clapham et al. 1999, Knowlton \& Kraus 2001). An overall low reproductive rate coupled with highly variable annual calving numbers represent further impediments to the recovery of this species (Kraus et al. 2001, 2007). Reproductive abnormalities were especially pronounced during the 1990s when the interval between calving for mature females increased from about 3.5 to almost $6 \mathrm{yr}$ and the age of first parturition also increased. Although causes of the apparent reproductive dysfunction in E. glacialis are not clear, reproductive success may be affected by habitat loss or degradation, nutritional stress, low genetic variability, disease, and sublethal exposure to pollutants as well as natural toxins such as paralytic shellfish poisoning (PSP) and amnesic shellfish poisoning (ASP) toxins produced by marine microalgae (Reeves et al. 2001).

ASP is a syndrome resulting from human ingestion of seafood, primarily shellfish, contaminated with domoic acid (DA), a potent water-soluble neurotoxin produced by certain species of the diatom genus Pseudonitzschia. DA binds with high affinity to kainate subtypes of glutamate receptors and also activates $N$ methyl $D$-aspartate (NMDA) ionotropic receptors, leading to neuronal excitotoxicity and degeneration (Berman et al. 2002, Ramsdell 2007). The first and only reported human ASP incident occurred in 1987 when over 100 people were poisoned by eating DA-contaminated mussels from Prince Edward Island, Canada (Perl et al. 1990). Many marine mammal and bird deaths have also been attributed to DA exposure through trophic transfer of the toxin via vector species. In 1991 over 145 brown pelicans Pelicanus occidentalis and Brandt's cormorants Phalacrocorax penicillatus died in Monterey Bay, CA, USA, after ingesting DAcontaminated northern anchovies Engraulis mordax (Work et al. 1993). In 1998 during a Pseudo-nitzschia australis bloom off the California coast, over 400 California sea lions Zalophus californianus stranded after being exposed to DA via ingestion of anchovies and sardines (Lefebvre et al. 1999, Scholin et al. 2000). Since then, many smaller scale mortality events associated with DA and involving mostly sea lions, but also other pinnipeds and cetaceans, have continued to occur in this region (reviewed by Van Dolah 2005).

Recent work has indicated that DA exposure in California sea lions resulted in reproductive failure in 209 stranded females, with symptoms including abortion, death in utero, and premature parturition (Brodie et al. 2006). Moreover, even low levels of exposure to this toxin have been proposed to cause fetal poisoning that manifests as neurological disease in adult animals later in life (Ramsdell \& Zabka 2008). In light of the apparent reproductive abnormalities in Eubalaena glacialis (Kraus et al. 2007) and the well-documented occurrence of DA-producing Pseudo-nitzschia spp. on their spring and summer feeding grounds (Kaczmarska et al. 2005), it is reasonable to suspect that these right whales may be exposed to this algal biotoxin.

During the summer months, Eubalaena glacialis migrate to the waters of the Gulf of Maine, USA, and Bay of Fundy, Canada, to feed almost exclusively on the predominant marine copepod, Calanus finmarchicus (Murison \& Gaskin 1989, Kenney et al. 2001, Baumgartner et al. 2003a,b, Baumgartner \& Mate 2003, 2005). Recently, Durbin et al. (2002) and Doucette et al. (2006) demonstrated that E. glacialis was exposed to PSP toxins by ingesting contaminated $C$. finmarchicus; nevertheless, to date there have been no investigations of North Atlantic right whale exposure to domoic acid. The primary objective of this study was to assess the presence of DA in North Atlantic right whale feces while on their feeding grounds. This information will aid in establishing the risk of E. glacialis exposure to this potent neurotoxin, as well as providing a foundation for further investigation of its potential effects on right whale health and reproduction. Our work was also aimed at establishing a possible route(s) for DA trophic transfer from its algal producers to right whales and identifying Pseudo-nitzschia species serving as the possible toxin source(s).

\section{MATERIALS AND METHODS}

Fecal sample collection. North Atlantic right whale fecal samples were collected in proximity to feeding whales in 2005 and 2006 during cruises covering the Great South Channel, Bay of Fundy, and Roseway Basin areas of the western North Atlantic (Fig. 1). Floating fecal samples were located opportunistically during boat-based population surveys and by directed fecal sampling surveys using a scent detection dog trained to target right whale feces (Rolland et al. 2006). Samples were harvested using a custom-made $300 \mu \mathrm{m}$ mesh net attached to a pole (Sea-Gear). Sub-samples were placed in $50 \mathrm{ml}$ polypropylene centrifuge tubes on ice and stored frozen $\left(-20^{\circ} \mathrm{C}\right)$ upon return to the field station until shipped overnight on dry ice to the NOAA Charleston Laboratory (Charleston, SC). Fecal material from one Cape Cod Bay (1999) and one Great South Channel (2005) stranded right whale (Fig. 1) were provided by Dr. M. Moore (Woods Hole Oceanographic Institution, Woods Hole, MA, USA). Aliquots of all fecal samples were shipped in the above manner to the NOAA Charleston Laboratory and stored at $-80^{\circ} \mathrm{C}$ until analyzed.

Plankton collection. Zooplankton samples (mostly copepods) were harvested in the vicinity of sighted Eubalaena glacialis using $333 \mu \mathrm{m}$ mesh bongo nets during 2 separate research cruises conducted in the 


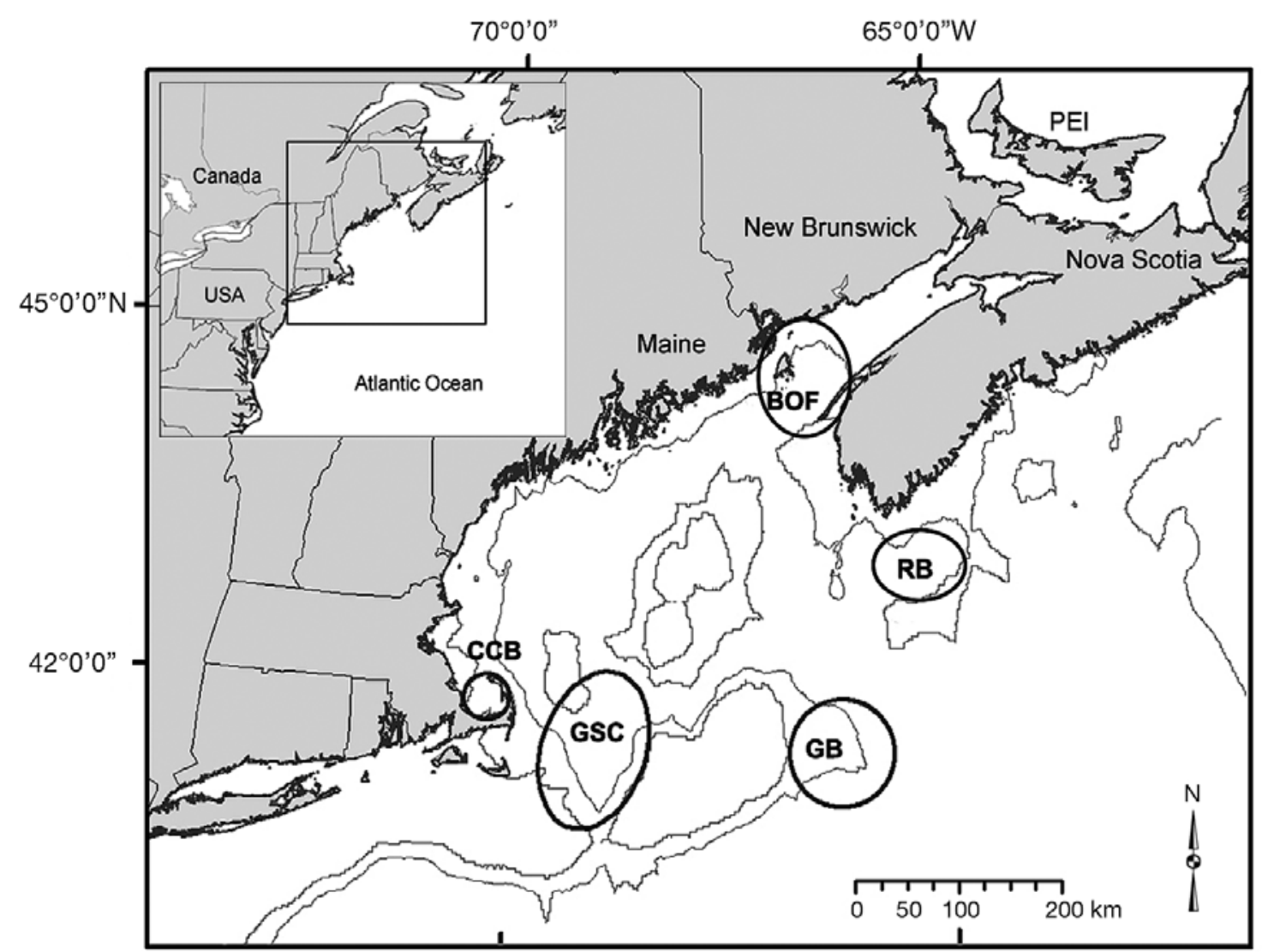

Fig. 1. Zooplankton and fecal sample collection sites: Bay of Fundy (BOF), Roseway Basin (RB), Georges Bank (GB), Great South Channel (GSC), and Cape Cod Bay (CCB). Circles denote sample collection perimeters

Bay of Fundy, Roseway Basin, and Georges Bank areas (Fig. 1) from July through September 2005 (n=11) and 2006 ( $\mathrm{n}=27$ ) by Drs. T. Cole (National Marine Fisheries Service, Woods Hole, MA, USA) and H. Koopman (Univ. of N. Carolina, Wilmington, NC, USA), respectively. Bongo nets were towed obliquely from surface down to approximately $200 \mathrm{~m}$ in depth for 3 to $19 \mathrm{~min}$. Material was filtered through a sieve (333 $\mu \mathrm{m}$ mesh), frozen on site $\left(-10^{\circ} \mathrm{C}\right)$, and later sent to the NOAA Charleston Laboratory where they were stored at $-80^{\circ} \mathrm{C}$ until analyzed.

Phytoplankton samples for species identification were collected simultaneously with fecal samples in the Bay of Fundy (August through September 2006; n = 10) using a $10 \mu \mathrm{m}$ mesh plankton net (Sea-Gear). Vertical net tows were performed down to a depth of $5 \mathrm{~m}$ and contents preserved in $2 \%$ acidified formalin $11: 1$ ratio of formalin:acetic acid) until examined by electron microscopy (EM). Surface bucket samples $(\mathrm{n}=10)$ were collected concurrently with net tows for total Pseudo-nitzschia cell counts and toxin analysis. Briefly, seawater samples (4 to $8 \mathrm{l}$ ) were passed through a custom-made sieve (10 $\mu \mathrm{m}$ mesh), preserved in $2 \%$ acidified formalin and refrigerated until examined by light microscopy. Subsets of each seawater sample (300 to
$600 \mathrm{ml}$ ) were filtered through $47 \mathrm{~mm}$ diam GF/F glass fiber filters for toxin analysis. Filters were placed immediately in cryovials, frozen in liquid nitrogen vapor, and later shipped on dry ice to the NOAA Charleston Laboratory where they were stored $\left(-80^{\circ} \mathrm{C}\right)$ until analyzed. Surface seawater samples $(\mathrm{n}=11)$ were collected in 2005 by Dr. T. Cole (July and August) as described previously and used for Pseudo-nitzschia spp. identification and enumeration by electron and light microscopy.

Toxin extraction. DA was extracted from fecal samples as described in Lefebvre et al. (2002) with several modifications. A 1:4 ratio (w/v) of each fecal sample (2 to $4 \mathrm{~g}$ ) to extraction solvent (50\% aqueous methanol) was sonicated (450 W; Sonifier S-450A; Branson Ultrasonics) on ice for 2 min and centrifuged (IEC Multi RF; Thermo Electron) at $5500 \times g$ for $10 \mathrm{~min}$. The resulting supernatants were filtered through $0.45 \mu \mathrm{m}$ followed by $0.22 \mu \mathrm{m}$ hydrophilic polypropylene (GHP) filter disks (Acrodisc; Pall Life Sciences) into $5 \mathrm{ml}$ glass vials and stored frozen $\left(-20^{\circ} \mathrm{C}\right)$.

Extraction of DA from phytoplankton samples followed the method outlined in Scholin et al. (2000): GF/F filters were submerged in $4 \mathrm{ml}$ of $10 \%$ aqueous methanol in glass extraction tubes and ground with a 
Teflon pestle at room temperature for $1 \mathrm{~min}$ (250 rpm). The resulting homogenate was centrifuged for $1 \mathrm{~min}$ at $52 \times g$ (IEC Centra GP8R; Thermo Electron) and sonicated $(450 \mathrm{~W})$ on an ice slurry for an additional $1 \mathrm{~min}$. Extracts were centrifuged for $1 \mathrm{~min}$ at $5500 \times g$ and supernatants filtered through $0.22 \mu \mathrm{m}$ GHP filter disks and stored at $-80^{\circ} \mathrm{C}$.

DA was extracted from zooplankton using a modified version of the above protocols. Briefly, copepod samples $(\sim 1 \mathrm{~g})$ were combined 1:4 (w/v) with 50\% aqueous methanol. Mixtures were homogenized with a Teflon pestle on ice, sonicated $(450 \mathrm{~W})$ for $2 \mathrm{~min}$, and extracts were then centrifuged, filtered, and stored as described above for fecal samples. When euphausiids (i.e. krill) were present in zooplankton samples, these animals were extracted separately from the copepods following this same procedure.

Toxin analysis. DA was measured in phytoplankton, zooplankton, and fecal sample extracts using a surface plasmon resonance (SPR) optical immunosensor (Biacore Q; GE Healthcare-Biacore), based on a protocol modified from Traynor et al. (2006). Zooplankton and fecal extracts were mixed 9:1 (v/v) with non-specific binding buffer (NSB; GE Healthcare-Biacore) to reduce sample matrix effects, diluted to $10 \%$ aqueous methanol in $0.2 \mu \mathrm{m}$-filtered and degassed HBS-EP buffer (GE Healthcare-Biacore). All extracts were further diluted in $10 \% \mathrm{MeOH}$ (degassed) as necessary for quantification based on a calibration curve comprising a DA certified reference standard $\left(1 \times 10^{4}\right.$ to $1 \times 10^{-3} \mathrm{ng}$ $\mathrm{DA} \mathrm{ml}^{-1}$; Institute for Marine Biosciences, Halifax, NS, Canada). Approximately $220 \mu \mathrm{l}$ of each sample, standard, Quality Control (QC) (1 ng DA ml-1), and blank $(10 \% \mathrm{MeOH})$ were mixed 9:1 (v/v) with a rabbit polyclonal anti-DA antibody (diluted previously 1:137.5 (v/v) in HBS-EP buffer) in a 96-well plate. A $150 \mu \mathrm{l}$ aliquot of each sample/antibody mixture was injected at a rate of $25 \mu \mathrm{min}^{-1}$ over a CM5 sensor chip (GE Healthcare-Biacore) surface upon which DA was immobilized. The amount of antibody bound to the chip surface, which is inversely proportional to the sample DA concentration, was measured as relative resonance units. DA concentrations in sample extracts were estimated on the basis of the above calibration curve with data processed using GraphPad Prism (ver 4; GraphPad Software).

A subset of fecal and zooplankton extracts was also tested for the presence of DA using a microplate receptor binding assay (RBA; Van Dolah et al. 1997) as described in Lefebvre et al. (1999) with some modifications: $12 \mu \mathrm{l}$ of sample were mixed with $12 \mu \mathrm{l}$ of glutamate decarboxylase (GAD; 100 units $\mathrm{ml}^{-1}$ ) and $96 \mu \mathrm{l}$ of GAD buffer (50 mM sodium acetate, $\mathrm{pH} 4.6 ; 2 \mathrm{mM}$ pyroxidoxal-5-phosphate; $100 \mathrm{mM}$ sodium chloride), and held for $30 \mathrm{~min}$ at room temperature to remove ambient glutamate. Each well of a 96-well filtration plate (MultiScreen; Millipore) then received sequentially $35 \mu \mathrm{l}$ of $50 \mathrm{mM}$ Tris buffer ( $\mathrm{pH} 7.4$ ), $35 \mu \mathrm{l}$ of sample, DA standard $\left(10^{-6}\right.$ to $10^{-11} \mathrm{M}$; Institute for Marine Biosciences, Halifax, NS, Canada) or QC (5 nM DA standard), $35 \mu \mathrm{l}$ of $5 \mathrm{nM}$ tritiated kainic acid (New England Nuclear), and $105 \mu \mathrm{l}$ of Sf9 insect membrane prep (Cell Trends). After $1 \mathrm{~h}$ incubation at $4^{\circ} \mathrm{C}$, each well was rinsed twice with $200 \mu$ l of cold Tris buffer (50 mM, $\mathrm{pH} 4.7$ ), followed by the addition of $50 \mu$ l of OptiPhase liquid scintillant (PE-Wallac) and incubation for $30 \mathrm{~min}$ at room temperature. Plates were counted on a microplate scintillation counter (Microbeta 1450, PEWallac) and DA concentrations in samples estimated on the basis of the above calibration curve using the MultiCalc software (ver 2.70.004; PE-Wallac).

Selected fecal and zooplankton extracts were analyzed further by liquid chromatography coupled with tandem mass spectrometry (LC-MS/MS) using an HP1100 LC system (Agilent Technologies) and an Applied Biosystems/MDS Sciex API 4000 triple quadruple mass spectrometer equipped with a Turbo $\mathrm{V}^{\mathrm{TM}}$ source (Applied Biosystems), as described by Wang et al. (2007). LC separation was performed on a Luna C18 (2) column $(150 \times 2 \mathrm{~mm}, 5 \mu \mathrm{m}$; Phenomenex $)$ using a gradient of water (A)/acetonitrile (B) containing $0.1 \%$ formic acid additive: 2 min of $5 \% \mathrm{~B}$, linear gradient to $40 \% \mathrm{~B}$ at $15 \mathrm{~min}, 95 \% \mathrm{~B}$ at $17 \mathrm{~min}$, held for $5 \mathrm{~min}$, then returned to initial conditions at $23 \mathrm{~min}$ and held for $5 \mathrm{~min}$ before the next injection. The injection volume was $5 \mu \mathrm{l}$ and the flow rate was $0.2 \mathrm{ml} \mathrm{min}^{-1}$. A diverter valve was used to divert the LC eluent before and after the elution of DA into waste to reduce contamination. DA detection was achieved by multiple reaction monitoring (MRM) using the $3 \mathrm{MRM}$ transitions from the protonated DA ion: $\mathrm{m} / \mathrm{z} 312 \rightarrow 266, \mathrm{~m} / \mathrm{z} 312 \rightarrow$ 248 , and $m / z 312 \rightarrow 193$. Quantification was based on a DA certified reference standard (Institute for Marine Biosciences).

Microscopic analyses. Phytoplankton samples collected in 2005 and $2006(\mathrm{n}=21$ ) were examined under a light microscope (Olympus BX51; Olympus America) and total Pseudo-nitzschia cell counts performed using a $1 \mathrm{ml}$ Sedgewick Rafter counting chamber (Hauser Scientific Partnership). Samples containing low Pseudo-nitzschia cell numbers $\left(\leq 1000\right.$ cells $\left.\mathrm{l}^{-1}\right)$ were concentrated further by centrifugation ( $5 \mathrm{~min}_{i} 1000 \times$ $g$ ), pipetting off a known volume of supernatant, and re-suspending cells in the remaining seawater, before counting.

Selected fecal samples were examined by scanning electron microscopy (SEM) to identify potentially toxic Pseudo-nitzschia spp. frustules $(\mathrm{n}=21)$ and copepod mouth parts $(\mathrm{n}=20)$. Phytoplankton samples $(\mathrm{n}=16)$ were also analyzed by SEM to determine the presence 
and identity of Pseudo-nitzschia spp. in the water column. Sample preparation followed a modification of the protocol given in Lefebvre et al. (2002). A few drops of fecal material were oxidized in glass test tubes by treating with an equivalent volume of $10 \%$ aqueous $\mathrm{KMnO}_{4}$ for $24 \mathrm{~h}$ at room temperature. Each sample was then mixed 1:3 (v/v) with $12 \mathrm{M} \mathrm{HCl}$ and boiled in a water bath for $1 \mathrm{~h}$ or until bleached. Five to twenty drops of acidified sample were pipetted onto $2 \mu \mathrm{m}$, $25 \mathrm{~mm}$ diameter GE PCTE (polycarbonate) filters (GE Osmonics) and rinsed under low vacuum with $12 \mathrm{ml}$ of Milli-Q water, $4 \mathrm{ml}$ each of a graded acetone series (10 to $80 \%$ ), followed by $8 \mathrm{ml}$ of $100 \%$ acetone, and $1 \mathrm{ml}$ of hexamethyldisilizane (HMDS). For analysis of copepod remnants, 1 to 2 drops of feces were pipetted directly onto $5 \mu \mathrm{m}, 25 \mathrm{~mm}$ diameter polycarbonate filters (GE Osmonics) and rinsed as outlined above. Phytoplankton samples $(5 \mathrm{ml})$ were mixed with $1.5 \mathrm{ml}$ of $10 \%$ aq. $\mathrm{KMnO}_{4}$, digested for $24 \mathrm{~h}$, and bleached with an equal volume of $\mathrm{HCl}$ as described above. One to five $\mathrm{ml}$ of acidified material were filtered through $5 \mu \mathrm{m}, 25 \mathrm{~mm}$ diameter polycarbonate filters and rinsed as described for fecal samples. All filters were air-dried for $\sim 5 \mathrm{~min}$, placed onto $25 \mathrm{~mm}$ diameter SEM stubs (Electron Microscopy Sciences), and sputter-coated with gold-palladium prior to examination by SEM (JEOL JSM 5600 LV; Jeol USA).

Selected phytoplankton $(n=2)$ and fecal $(n=4)$ samples were analyzed further by transmission electron microscopy (TEM). Sample cleanup was performed as described above for SEM analysis. Cleaned material was rinsed 3 times with $20 \mathrm{ml}$ of Milli-Q water through a $0.2 \mu \mathrm{m}$ pore size, $47 \mathrm{~mm}$ diameter polycarbonate filter (GE Osmonics), re-suspended in $5 \mathrm{ml}$ of Milli-Q water, and kept refrigerated until used. Several drops of cleaned material were placed onto formvar-coated copper grids, dried, and viewed in a JEM-1010 transmission electron microscope (Jeol). Morphometric data obtained from SEM and TEM micrographs of Pseudonitzschia spp. frustules were used for species identification and compared to Pseudo-nitzschia spp. micrographs published by Skov et al. (1999), Lundholm et al. (2003, 2006), and Kaczmarska et al. (2005). For species determination of frustule fragments present in whale feces, striae and fibulae counts were extrapolated from a $5 \mu \mathrm{m}$ section of the available frustule.

Table 1. Eubalaena glacialis. Domoic acid (DA) levels in right whale feces collected in 1999 and 2005-2006 from Cape Cod Bay (CCB), Great South Channel (GSC), Roseway Basin (RB), and Bay of Fundy (BOF) areas, as measured by surface plasmon resonance (SPR), receptor binding assay (RBA), and liquid chromatography-tandem mast spectrometry (LC-MS/MS). <DL: samples for which DA levels were below a method's detection limit (DLs for SPR, RBA, and LC-MS/MS = 0.01, 0.09, and 0.002 $\mu \mathrm{g}$ DA $\mathrm{g}^{-1}$, respectively). nd: no available latitude/longitude data

\begin{tabular}{|c|c|c|c|c|c|c|c|}
\hline \multirow{2}{*}{$\begin{array}{l}\text { Sample } \\
\text { ID }\end{array}$} & \multirow{2}{*}{$\begin{array}{l}\text { Latitude } \\
\qquad\left({ }^{\circ} \mathrm{N}\right)\end{array}$} & \multirow{2}{*}{$\begin{array}{l}\text { Longitude } \\
\left({ }^{\circ} \mathrm{W}\right)\end{array}$} & \multirow{2}{*}{$\begin{array}{l}\text { Sample } \\
\text { location }\end{array}$} & \multirow{2}{*}{$\begin{array}{l}\text { Sample date } \\
\text { (mm/dd/yy) }\end{array}$} & \multicolumn{3}{|c|}{ DA conc. in whale feces ( $\mu \mathrm{g} \mathrm{DA} \mathrm{g}{ }^{-1}$ feces) } \\
\hline & & & & & SPR & RBA & LC-MS/MS \\
\hline $06-001^{\mathrm{a}}$ & $41^{\circ} 54.3^{\prime}$ & $70^{\circ} 08.9^{\prime}$ & $\mathrm{CCB}$ & $04 / 21 / 99$ & 0.02 & 0.14 & 0.003 \\
\hline $05-001^{a}$ & nd & nd & GSC & 04/29/05 & 0.34 & 0.10 & 0.021 \\
\hline 05-103 & $41^{\circ} 08.7^{\prime}$ & $69^{\circ} 14.5^{\prime}$ & GSC & 05/18/05 & 0.19 & 0.15 & 0.062 \\
\hline 05-104 & $41^{\circ} 08.8^{\prime}$ & $69^{\circ} 14.7^{\prime}$ & GSC & 05/18/05 & 0.23 & 0.15 & 0.113 \\
\hline 05-002 & $42^{\circ} 02.7^{\prime}$ & $68^{\circ} 54.0^{\prime}$ & GSC & 06/09/05 & 0.61 & 0.18 & 0.175 \\
\hline 05-007 & $44^{\circ} 36.4^{\prime}$ & $66^{\circ} 25.8^{\prime}$ & $\mathrm{BOF}$ & 08/12/05 & $<\mathrm{DL}$ & $<\mathrm{DL}$ & 0.002 \\
\hline 05-019 & $44^{\circ} 37.7^{\prime}$ & $66^{\circ} 25.0^{\prime}$ & BOF & 08/19/05 & 0.02 & $<\mathrm{DL}$ & 0.004 \\
\hline 05-028 & $44^{\circ} 40.4^{\prime}$ & $66^{\circ} 22.9^{\prime}$ & BOF & 08/24/05 & 0.22 & 0.11 & 0.016 \\
\hline 05-029 & $44^{\circ} 40.2^{\prime}$ & $66^{\circ} 23.3^{\prime}$ & BOF & 08/24/05 & 0.26 & 0.13 & 0.012 \\
\hline 05-093 & $44^{\circ} 36.5^{\prime}$ & $66^{\circ} 30.2^{\prime}$ & BOF & 08/25/05 & 0.06 & $<\mathrm{DL}$ & 0.008 \\
\hline 05-031 & $44^{\circ} 38.4^{\prime}$ & $66^{\circ} 25.9^{\prime}$ & $\mathrm{BOF}$ & $09 / 02 / 05$ & 0.07 & $<\mathrm{DL}$ & 0.035 \\
\hline 05-032 & $44^{\circ} 39.9^{\prime}$ & $66^{\circ} 24.6^{\prime}$ & $\mathrm{BOF}$ & 09/02/05 & 0.13 & $<\mathrm{DL}$ & 0.026 \\
\hline 05-038 & $44^{\circ} 41.0^{\prime}$ & $66^{\circ} 21.7^{\prime}$ & BOF & 09/04/05 & 0.15 & 0.10 & 0.008 \\
\hline 05-048 & $44^{\circ} 38.3^{\prime}$ & $66^{\circ} 25.0^{\prime}$ & $\mathrm{BOF}$ & $09 / 05 / 05$ & 0.14 & $<\mathrm{DL}$ & 0.014 \\
\hline 05-057 & $44^{\circ} 36.7^{\prime}$ & $66^{\circ} 26.8^{\prime}$ & BOF & 09/06/05 & 0.32 & 0.59 & 0.026 \\
\hline 05-060 & $44^{\circ} 37.7^{\prime}$ & $66^{\circ} 25.8^{\prime}$ & $\mathrm{BOF}$ & 09/06/05 & 0.04 & $<\mathrm{DL}$ & 0.009 \\
\hline 05-081 & $44^{\circ} 38.0^{\prime}$ & $66^{\circ} 29.2^{\prime}$ & $\mathrm{BOF}$ & 09/13/05 & 0.03 & $<\mathrm{DL}$ & 0.004 \\
\hline 06-002 & $44^{\circ} 39.5^{\prime}$ & $66^{\circ} 31.1^{\prime}$ & $\mathrm{BOF}$ & 08/04/06 & 0.35 & 0.17 & 0.171 \\
\hline 06-012 & $44^{\circ} 37.6^{\prime}$ & $66^{\circ} 24.7^{\prime}$ & $\mathrm{BOF}$ & 08/27/06 & 0.13 & 0.10 & 0.068 \\
\hline 06-020 & $44^{\circ} 37.7^{\prime}$ & $66^{\circ} 22.7^{\prime}$ & BOF & 09/07/06 & 0.07 & 0.10 & 0.023 \\
\hline 06-023 & $44^{\circ} 40.1^{\prime}$ & $66^{\circ} 27.0^{\prime}$ & BOF & 09/17/06 & 0.19 & 0.15 & 0.084 \\
\hline 06-028 & $42^{\circ} 53.7^{\prime}$ & $65^{\circ} 09.6^{\prime}$ & $\mathrm{RB}$ & 09/17/06 & 0.02 & 0.11 & $<\mathrm{DL}$ \\
\hline 06-029 & $42^{\circ} 55.8^{\prime}$ & $65^{\circ} 08.3^{\prime}$ & $\mathrm{RB}$ & 09/18/06 & 0.02 & 0.15 & $<\mathrm{DL}$ \\
\hline 06-035 & $44^{\circ} 64.9^{\prime}$ & $66^{\circ} 42.2^{\prime}$ & BOF & 09/28/06 & 0.03 & 0.12 & 0.007 \\
\hline
\end{tabular}


Table 2. Domoic acid (DA) levels in krill and copepods collected in 2005-2006 from Georges Bank (GB), Roseway Basin (RB), and Bay of Fundy (BOF) areas, as measured by surface plasmon resonance (SPR), receptor binding assay (RBA), and liquid chromatography-tandem mass spectrometry (LC-MS/MS). <DL: samples for which DA levels were below a method's detection limit

(DLs for: SPR, RBA, and LC-MS/MS = 0.01, 0.09, and $0.002 \mu \mathrm{gA} \mathrm{g}^{-1}$, respectively)

\begin{tabular}{|c|c|c|c|c|c|c|c|c|}
\hline \multirow{2}{*}{$\begin{array}{l}\text { Sample } \\
\text { ID }\end{array}$} & \multirow{2}{*}{$\begin{array}{l}\text { Sample } \\
\text { type }\end{array}$} & \multirow{2}{*}{$\begin{array}{l}\text { Lat. } \\
\left({ }^{\circ} \mathrm{N}\right)\end{array}$} & \multirow{2}{*}{$\begin{array}{l}\text { Long. } \\
\left({ }^{\circ} \mathrm{W}\right)\end{array}$} & \multirow{2}{*}{$\begin{array}{l}\text { Sample } \\
\text { location }\end{array}$} & \multirow{2}{*}{$\begin{array}{l}\text { Sample date } \\
\text { (mm/dd/yy) }\end{array}$} & \multicolumn{3}{|c|}{ DA conc. in zooplankton ( $\mu \mathrm{g} \mathrm{DA} \mathrm{g}^{-1}$ tissue) } \\
\hline & & & & & & SPR & RBA & LC-MS/MS \\
\hline Z05-10 & Krill & $42^{\circ} 05.1^{\prime}$ & $65^{\circ} 57.2^{\prime}$ & GB & $07 / 31 / 05$ & 0.04 & $<\mathrm{DL}$ & $<\mathrm{DL}$ \\
\hline Z05-32 & Copepod & $42^{\circ} 51.4^{\prime}$ & $65^{\circ} 27.1^{\prime}$ & $\mathrm{RB}$ & 08/01/05 & 0.03 & $<\mathrm{DL}$ & $<\mathrm{DL}$ \\
\hline Z05-50 & Copepod & $44^{\circ} 35.5^{\prime}$ & $66^{\circ} 27.3^{\prime}$ & BOF & 08/04/05 & 0.03 & $<\mathrm{DL}$ & $<\mathrm{DL}$ \\
\hline Z05-63 & Copepod & $41^{\circ} 31.6^{\prime}$ & $66^{\circ} 01.0^{\prime}$ & GB & 08/06/05 & 0.03 & $<\mathrm{DL}$ & $<\mathrm{DL}$ \\
\hline Z05-64 & Copepod & $41^{\circ} 29.6^{\prime}$ & $66^{\circ} 01.9^{\prime}$ & GB & 08/06/05 & 0.05 & $<\mathrm{DL}$ & $<\mathrm{DL}$ \\
\hline Z05-81a & Copepod & $41^{\circ} 33.6^{\prime}$ & $66^{\circ} 01.8^{\prime}$ & GB & 08/06/05 & 0.06 & $<\mathrm{DL}$ & 0.004 \\
\hline Z05-81b & Krill & $41^{\circ} 33.6^{\prime}$ & $66^{\circ} 01.8^{\prime}$ & GB & 08/06/05 & 0.07 & $<\mathrm{DL}$ & 0.004 \\
\hline Z05-128b & Krill & $44^{\circ} 34.9^{\prime}$ & $66^{\circ} 27.6^{\prime}$ & BOF & 08/15/05 & 0.03 & $<\mathrm{DL}$ & $<\mathrm{DL}$ \\
\hline Z06-1 & Copepod & $44^{\circ} 40.0^{\prime}$ & $66^{\circ} 24.0^{\prime}$ & BOF & 07/20/06 & 0.04 & $<\mathrm{DL}$ & 0.005 \\
\hline Z06-2 & Copepod & $44^{\circ} 34.0^{\prime}$ & $66^{\circ} 25.0^{\prime}$ & BOF & 07/20/06 & 0.06 & $<\mathrm{DL}$ & 0.011 \\
\hline Z06-7 & Copepod & $44^{\circ} 35.0^{\prime}$ & $66^{\circ} 25.0^{\prime}$ & BOF & 08/12/06 & 0.10 & $<\mathrm{DL}$ & 0.033 \\
\hline Z06-10 & Copepod & $44^{\circ} 39.9^{\prime}$ & $66^{\circ} 35.9^{\prime}$ & BOF & 08/23/06 & 0.05 & $<\mathrm{DL}$ & 0.013 \\
\hline Z06-12 & Copepod & $44^{\circ} 30.1^{\prime}$ & $66^{\circ} 19.0^{\prime}$ & BOF & 08/24/06 & 0.06 & $<\mathrm{DL}$ & 0.010 \\
\hline Z06-13 & Copepod & $44^{\circ} 30.2^{\prime}$ & $66^{\circ} 27.3^{\prime}$ & BOF & 08/24/06 & 0.05 & $<\mathrm{DL}$ & 0.009 \\
\hline Z06-16b & Krill & $44^{\circ} 34.8^{\prime}$ & $66^{\circ} 25.0^{\prime}$ & BOF & 09/02/06 & 0.06 & $<\mathrm{DL}$ & 0.012 \\
\hline Z06-17 & Copepod & $44^{\circ} 35.1^{\prime}$ & $66^{\circ} 25.0^{\prime}$ & $\mathrm{BOF}$ & 09/07/06 & 0.17 & $<\mathrm{DL}$ & 0.054 \\
\hline Z06-22 & Copepod & $44^{\circ} 35.2^{\prime}$ & $66^{\circ} 27.3^{\prime}$ & $\mathrm{BOF}$ & 09/27/06 & 0.02 & $<\mathrm{DL}$ & 0.002 \\
\hline
\end{tabular}

\section{RESULTS}

\section{Toxin analyses}

All but one of the Eubalaena glacialis fecal samples from 2005 ( $\mathrm{n}=$ 46) and all samples from $2006(n=24)$ tested positive for the presence of DA by SPR, with concentrations ranging from 0.02 to 0.61 and 0.02 to $0.35 \mu \mathrm{g}$ DA $\mathrm{g}^{-1}$ feces, respectively. The average DA concentration in 2005 Bay of Fundy samples (August through September) was $0.08 \pm 0.07 \mu \mathrm{g} \mathrm{DA} \mathrm{g}{ }^{-1}$ (mean $\pm \mathrm{SD} ; \mathrm{n}=40$ ) in contrast to $0.3 \pm$ $0.2 \mu \mathrm{g} \mathrm{DA} \mathrm{g}{ }^{-1}(\mathrm{n}=5)$ in Great South Channel samples (April through June). Average DA concentrations in 2006 fecal samples were $0.09 \pm 0.08 \mu \mathrm{g}$ DA $g^{-1}(n=20)$ for Bay of Fundy (August through September) and $0.02 \pm 0.01 \mu \mathrm{g} \mathrm{DA} \mathrm{g}^{-1}(\mathrm{n}=4)$ for Roseway Basin (September). Feces obtained from the 1999 and 2005 stranded North Atlantic right whales also contained DA (Table 1).

All $2005(\mathrm{n}=11)$ and $2006(\mathrm{n}=27)$ zooplankton (copepod and krill) samples tested positive for the presence of DA by SPR, with values ranging from
Table 3. Pseudo-nitzschia spp. present in surface phytoplankton samples collected in 2005-2006 from Bay of Fundy (BOF), Georges Bank (GB), and Roseway Basin (RB) areas. Total Pseudo-nitzschia cell counts did not exceed

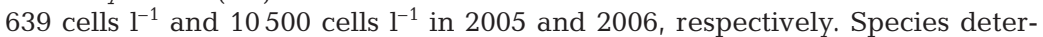
mination was performed by SEM and/or TEM. Species key: A $=P$. delicatissima $; \mathrm{B}=P$. pungens $; \mathrm{C}=P$. seriata $; \mathrm{E}=P$. fraudulenta $; \mathrm{F}=P$. subpacifica $;$ $\mathrm{G}=P$. turgidula $; \mathrm{I}=P$. cuspidata $\mathrm{J}=P$. pseudodelicatissima $; \mathrm{K}=P$. cf. calliantha; $\mathrm{U}=$ unidentifiable Pseudo-nitzschia spp.; $\mathrm{N}=$ no Pseudo-nitzschia spp. observed

\begin{tabular}{|c|c|c|c|c|c|}
\hline $\begin{array}{l}\text { Sample } \\
\text { ID }\end{array}$ & $\begin{array}{l}\text { Latitude } \\
\left({ }^{\circ} \mathrm{N}\right)\end{array}$ & $\begin{array}{l}\text { Longitude } \\
\left.\qquad{ }^{\circ} \mathrm{W}\right)\end{array}$ & $\begin{array}{l}\text { Sample } \\
\text { location }\end{array}$ & $\begin{array}{l}\text { Date collected } \\
\text { (mm/dd/yy) }\end{array}$ & $\begin{array}{c}\text { Phytoplankton } \\
\text { analyses }\end{array}$ \\
\hline P05-1 & $41^{\circ} 20.0^{\prime}$ & $66^{\circ} 08.0^{\prime}$ & GB & 07/29/05 & $\mathrm{U}^{\mathrm{a}}$ \\
\hline P05-10 & $42^{\circ} 05.1^{\prime}$ & $65^{\circ} 57.2^{\prime}$ & GB & $07 / 31 / 05$ & $\mathrm{U}^{\mathrm{a}}$ \\
\hline P05-31 & $42^{\circ} 52.6^{\prime}$ & $65^{\circ} 16.7^{\prime}$ & $\mathrm{RB}$ & 08/01/05 & $\mathrm{A}, \mathrm{G}, \mathrm{I}, \mathrm{J}, \mathrm{K}$ \\
\hline P05-32 & $42^{\circ} 51.4^{\prime}$ & $65^{\circ} 27.1^{\prime}$ & $\mathrm{RB}$ & 08/01/05 & $\mathrm{U}^{\mathrm{a}}$ \\
\hline P05-50 & $44^{\circ} 35.5^{\prime}$ & $66^{\circ} 27.3^{\prime}$ & $\mathrm{BOF}$ & 08/04/05 & $\mathrm{C}$ \\
\hline P05-63 & $41^{\circ} 31.6^{\prime}$ & $66^{\circ} 01.0^{\prime}$ & GB & 08/06/05 & $\mathrm{U}^{\mathrm{a}}$ \\
\hline P05-64 & $41^{\circ} 29.6^{\prime}$ & $66^{\circ} 01.9^{\prime}$ & GB & 08/06/05 & $\mathrm{N}$ \\
\hline P05-81 & $41^{\circ} 33.6^{\prime}$ & $66^{\circ} 01.8^{\prime}$ & GB & 08/06/05 & B \\
\hline P05-82 & $41^{\circ} 33.7^{\prime}$ & $65^{\circ} 58.9^{\prime}$ & GB & 08/08/05 & $\mathrm{N}$ \\
\hline P05-128 & $44^{\circ} 34.9^{\prime}$ & $66^{\circ} 27.6^{\prime}$ & BOF & 08/15/05 & $\mathrm{A}, \mathrm{C}, \mathrm{E}, \mathrm{G}, \mathrm{I}, \mathrm{J}$ \\
\hline P05-130 & $44^{\circ} 39.7^{\prime}$ & $66^{\circ} 28.8^{\prime}$ & BOF & 08/15/05 & $\mathrm{U}^{\mathrm{a}}$ \\
\hline P06-1 & $44^{\circ} 39.5^{\prime}$ & $66^{\circ} 22.9^{\prime}$ & BOF & 08/30/06 & B \\
\hline P06-2 & $44^{\circ} 39.4^{\prime}$ & $66^{\circ} 26.1^{\prime}$ & BOF & 08/30/06 & $B, E, F$ \\
\hline P06-3 & $44^{\circ} 39.3^{\prime}$ & $66^{\circ} 29.5^{\prime}$ & BOF & 08/30/06 & $\mathrm{B}, \mathrm{F}$ \\
\hline P06-4 & $44^{\circ} 39.1^{\prime}$ & $66^{\circ} 28.4^{\prime}$ & BOF & 09/05/06 & $\mathrm{B}, \mathrm{F}$ \\
\hline P06-5 & $44^{\circ} 34.1^{\prime}$ & $66^{\circ} 31.3^{\prime}$ & BOF & 09/05/06 & B \\
\hline P06-6 & $44^{\circ} 32.5^{\prime}$ & $66^{\circ} 33.1^{\prime}$ & BOF & 09/05/06 & E \\
\hline P06-7 & $44^{\circ} 36.3^{\prime}$ & $66^{\circ} 34.8^{\prime}$ & $\mathrm{BOF}$ & 09/05/06 & $B, E, F$ \\
\hline P06-8 & $44^{\circ} 50.3^{\prime}$ & $66^{\circ} 58.5^{\prime}$ & BOF & 09/07/06 & $\mathrm{B}, \mathrm{E}, \mathrm{F}$ \\
\hline P06-9 & $44^{\circ} 39.7^{\prime}$ & $66^{\circ} 25.5^{\prime}$ & $\mathrm{BOF}$ & 09/07/06 & $\mathrm{B}, \mathrm{E}$ \\
\hline P06-10 & $44^{\circ} 37.5^{\prime}$ & $66^{\circ} 22.6^{\prime}$ & BOF & 09/07/06 & $\mathrm{B}, \mathrm{F}$ \\
\hline
\end{tabular}


Table 4. Pseudo-nitzschia spp. present in Eubalaena glacialis fecal samples collected in 2005-2006 from Bay of Fundy (BOF), Great South Channel (GSC), Cape Cod Bay (CCB), and Roseway Basin (RB) areas. Species determination was performed by SEM and/or TEM. Species key: $\mathrm{A}=P$. delicatissima; $\mathrm{B}=P$. pungens $; \mathrm{C}=P$. seriata $; \mathrm{D}=P$. pseudodelicatissima-complex $; \mathrm{E}=P$. fraudulenta $; \mathrm{F}=$ $P$. subpacifica; $\mathrm{G}=P$. cf. turgidula $\mathrm{H}=P$. multiseries $; \mathrm{L}=$ undescribed Pseudonitzschia spp.; $\mathrm{N}=$ no Pseudo-nitzschia spp. observed; nd: no available data

\begin{tabular}{|c|c|c|c|c|c|}
\hline $\begin{array}{l}\text { Sample } \\
\text { ID }\end{array}$ & $\begin{array}{l}\text { Latitude } \\
\left({ }^{\circ} \mathrm{N}\right)\end{array}$ & $\begin{array}{l}\text { Longitude } \\
\left({ }^{\circ} \mathrm{W}\right)\end{array}$ & $\begin{array}{l}\text { Sample } \\
\text { location }\end{array}$ & $\begin{array}{l}\text { Date collected } \\
\text { (mm/dd/yy) }\end{array}$ & $\begin{array}{c}\text { Fecal sample } \\
\text { analyses }\end{array}$ \\
\hline $06-001^{a}$ & $41^{\circ} 54.3^{\prime}$ & $70^{\circ} 08.9^{\prime}$ & $\mathrm{CCB}$ & 04/21/99 & $\mathrm{E}, \mathrm{D}^{\mathrm{b}}, \mathrm{H}$ \\
\hline $05-001^{a}$ & nd & nd & GSC & 04/29/05 & $A, D^{b}, C$ \\
\hline 05-103 & $41^{\circ} 08.7^{\prime}$ & $69^{\circ} 14.5^{\prime}$ & GSC & 05/18/05 & $\mathrm{A}, \mathrm{D}^{\mathrm{b}}$ \\
\hline 05-003 & $42^{\circ} 03.3^{\prime}$ & $68^{\circ} 55.1^{\prime}$ & GSC & 06/09/05 & G \\
\hline 05-018 & $44^{\circ} 37.5^{\prime}$ & $66^{\circ} 25.6^{\prime}$ & $\mathrm{BOF}$ & 08/19/05 & $\mathrm{A}, \mathrm{D}^{\mathrm{b}}$ \\
\hline $05-024$ & $44^{\circ} 36.4^{\prime}$ & $66^{\circ} 30.8^{\prime}$ & BOF & 08/19/05 & $\mathrm{D}^{\mathrm{b}}$ \\
\hline $05-028$ & $44^{\circ} 40.4^{\prime}$ & $66^{\circ} 22.9^{\prime}$ & $\mathrm{BOF}$ & $08 / 24 / 05$ & $\mathrm{D}^{\mathrm{b}}, \mathrm{C}, \mathrm{G}$ \\
\hline $05-031$ & $44^{\circ} 38.4^{\prime}$ & $66^{\circ} 25.9^{\prime}$ & BOF & 09/02/05 & $\mathrm{D}^{\mathrm{b}}, \mathrm{G}$ \\
\hline $05-038$ & $44^{\circ} 41.0^{\prime}$ & $66^{\circ} 21.7^{\prime}$ & BOF & 09/04/05 & $\mathrm{D}^{\mathrm{b}}$ \\
\hline $05-048$ & $44^{\circ} 38.3^{\prime}$ & $66^{\circ} 25.0^{\prime}$ & BOF & 09/05/05 & $\mathrm{N}$ \\
\hline $05-057$ & $44^{\circ} 36.7^{\prime}$ & $66^{\circ} 26.8^{\prime}$ & $\mathrm{BOF}$ & 09/06/05 & $\mathrm{A}, \mathrm{B}, \mathrm{D}^{\mathrm{b}}, \mathrm{G}, \mathrm{L}$ \\
\hline $05-060$ & $44^{\circ} 37.7^{\prime}$ & $66^{\circ} 25.8^{\prime}$ & $\mathrm{BOF}$ & 09/06/05 & $\mathrm{D}^{\mathrm{b}}, \mathrm{C}$ \\
\hline $05-064$ & $44^{\circ} 39.4^{\prime}$ & $66^{\circ} 24.0^{\prime}$ & BOF & 09/06/05 & $\mathrm{D}^{\mathrm{b}}$ \\
\hline $05-071$ & $44^{\circ} 40.1^{\prime}$ & $66^{\circ} 28.1^{\prime}$ & $\mathrm{BOF}$ & 09/13/05 & $\mathrm{N}$ \\
\hline 06-002 & $44^{\circ} 39.5^{\prime}$ & $66^{\circ} 31.1^{\prime}$ & $\mathrm{BOF}$ & 08/04/06 & B \\
\hline 06-012 & $44^{\circ} 37.6^{\prime}$ & $66^{\circ} 24.7^{\prime}$ & $\mathrm{BOF}$ & 08/27/06 & $\mathrm{B}, \mathrm{E}, \mathrm{F}$ \\
\hline 06-016 & $44^{\circ} 39.6^{\prime}$ & $66^{\circ} 21.1^{\prime}$ & $\mathrm{BOF}$ & 09/03/06 & $\mathrm{B}$ \\
\hline 06-023 & $44^{\circ} 40.1^{\prime}$ & $66^{\circ} 27.0^{\prime}$ & BOF & 09/17/06 & E, F \\
\hline $06-028$ & $42^{\circ} 53.7^{\prime}$ & $65^{\circ} 09.6^{\prime}$ & $\mathrm{RB}$ & 09/17/06 & $\mathrm{B}$ \\
\hline $06-035$ & $44^{\circ} 64.9^{\prime}$ & $66^{\circ} 42.2^{\prime}$ & $\mathrm{BOF}$ & 09/28/06 & $\mathrm{B}, \mathrm{E}$ \\
\hline $06-038$ & $44^{\circ} 70.3^{\prime}$ & $66^{\circ} 38.6^{\prime}$ & $\mathrm{BOF}$ & 09/28/06 & $\mathrm{B}, \mathrm{E}, \mathrm{F}$ \\
\hline \multicolumn{6}{|c|}{ 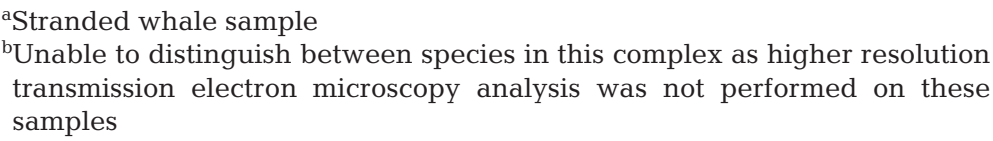 } \\
\hline
\end{tabular}

0.175 by LC-MS/MS (Table 1 ). Average DA concentrations in zooplankton were $0.06 \pm 0.04 \mu \mathrm{g} \mathrm{DA} \mathrm{g}{ }^{-1}(\mathrm{n}=17)$ and $0.01 \pm 0.02 \mu \mathrm{g} \mathrm{DA} \mathrm{g}{ }^{-1}(\mathrm{n}=11)$ by SPR and LC-MS/MS, respectively (Table 2). Values ranged from 0.02 to $0.17 \mu \mathrm{g} \mathrm{DA} \mathrm{g}^{-1}$ by SPR and from 0.002 to $0.054 \mu \mathrm{g} \mathrm{DA} \mathrm{g}^{-1}$ by LC-MS/MS (Table 2). DA levels in zooplankton were below the RBA limit of detection (0.09 $\left.\mu \mathrm{g} \mathrm{DA} \mathrm{g}^{-1}\right)$. Further LC-MS/MS analyses, with nuclear magnetic resonance (NMR) confirmation, revealed the presence of a putative methylated DA metabolite in zooplankton $(\mathrm{n}=17)$ and N. Atlantic right whale feces ( $\mathrm{n}=$ 17) (Z. Wang et al. unpubl. data).

\section{Microscopic analyses}

All phytoplankton samples examined by both light and electron microscopy, with the exception of sample ID numbers P05-64 and P05-82, contained cells of Pseudo-nitzschia spp. (Table 3). Total Pseudo-nitzschia cell counts in surface seawater samples ranged from 6 to 639 cells $\mathrm{l}^{-1}$ and from 663 to 10500 cells $\mathrm{l}^{-1}$ for $2005(\mathrm{n}=9$ ) and 2006 ( $\mathrm{n}=10)$, respectively (data not shown). Pseudo-nitzschia frustule fragments were present in all $(\mathrm{n}=21)$

0.03 to 0.07 and from 0.02 to $0.17 \mu \mathrm{g} \mathrm{DA} \mathrm{g}{ }^{-1}$ tissue, respectively (Table 2). Average zooplankton DA concentrations were $0.03 \pm 0.003 \mu \mathrm{g} \mathrm{DA} \mathrm{g}{ }^{-1}(\mathrm{n}=4)$ for 2005 Bay of Fundy (August), $0.03 \pm 0.01 \mu \mathrm{g} \mathrm{DA} \mathrm{g}^{-1}(\mathrm{n}=2)$ for Roseway Basin (August), $0.05 \pm 0.02 \mu \mathrm{g} \mathrm{DA} \mathrm{g}^{-1}(\mathrm{n}=5)$ for Georges Bank (July through August), and $0.04 \pm$ $0.03 \mu \mathrm{g} \mathrm{DA} \mathrm{g}^{-1}(\mathrm{n}=27)$ for all 2006 Bay of Fundy (July through September) samples. Average copepod ( $\mathrm{n}=$ $32)$ and krill $(n=6)$ DA concentrations were $0.04 \pm$ $0.03 \mu \mathrm{gA} \mathrm{g}^{-1}(\mathrm{n}=32)$ and $0.04 \pm 0.02 \mu \mathrm{g} \mathrm{DA} \mathrm{g}^{-1}(\mathrm{n}=$ 6), respectively. DA was not detected by SPR in any 2006 (August through September) Bay of Fundy phytoplankton samples (detection limit $0.01 \mu \mathrm{g} \mathrm{DA} \mathrm{g}{ }^{-1}$ ).

A subset of fecal $(n=24)$ and zooplankton $(n=17)$ samples tested by SPR was selected for comparative analysis by RBA and confirmation by LC-MS/MS (Tables $1 \& 2$ ). The average DA concentrations measured in feces by SPR, RBA, and LC-MS/MS were $0.16 \pm 0.15 \mu \mathrm{gA} \mathrm{g}^{-1}(\mathrm{n}=23), 0.16 \pm 0.12 \mu \mathrm{gA} \mathrm{g}^{-1}$ ( $\mathrm{n}=16)$, and $0.04 \pm 0.05 \mu \mathrm{g} \mathrm{DA} \mathrm{g}{ }^{-1}(\mathrm{n}=22)$, respectively (Table 1). Values ( $\mu$ g DA g ${ }^{-1}$ ) ranged from 0.02 to 0.61 by SPR, from 0.1 to 0.59 by RBA, and from 0.002 to but 2 (ID: 05-048 and 05-071) fecal samples analyzed (Table 4). Pseudo-nitzschia frustule measurements were taken from all available EM images and used for species identification (Table 5).

Pseudo-nitzschia seriata (Fig. 2B), P. delicatissima, and $P$. cf. turgidula (Fig. 2D) fragments were present in Great South Channel and Bay of Fundy fecal samples from April through June and from August through September of 2005, respectively (Table 4). P. cf. turgidula (Fig. 2D) fragments did not provide enough information for confident identification; nevertheless, the available morphometric data suggested that these fragments closely resembled $P$. turgidula (Table 5; Fig. 2D inset). The presence of $P$. seriata (Fig. 2B inset), $P$. delicatissima (Fig. $3 \mathrm{C}$ inset), and $P$. turgidula (Fig. 2D inset) was confirmed in one Bay of Fundy phytoplankton sample (P05-128) collected in August 2005, while the latter 2 Pseudo-nitzschia spp. were also seen in Roseway Basin phytoplankton sample ID number P05-31 earlier that month (Table 3). Several frustule fragments belonging to taxa within the P. pseudodelicatissima-complex, composed of P. pseudodelica- 
Table 5. Morphometric data (by transmission and scanning electron microscope [TEM and SEM]) for all Pseudo-nitzschia spp. present in fecal and phytoplankton samples. Values in parentheses indicate number of specimens analyzed. nd: no available data

\begin{tabular}{|c|c|c|c|c|c|c|c|c|c|c|}
\hline $\begin{array}{l}\text { Sample } \\
\text { type }\end{array}$ & $\begin{array}{l}\text { Valve } \\
\text { shape }\end{array}$ & $\begin{array}{l}\text { Length } \\
(\mu \mathrm{m})\end{array}$ & $\begin{array}{l}\text { Width } \\
(\mu \mathrm{m})\end{array}$ & $\begin{array}{l}\text { Central } \\
\text { nodule }\end{array}$ & $\begin{array}{l}\text { Striae per } \\
10 \mu \mathrm{m}\end{array}$ & $\begin{array}{l}\text { Fibulae } \\
\text { per } 10 \mu \mathrm{m}\end{array}$ & $\begin{array}{l}\text { Poroid } \\
\text { rows }\end{array}$ & $\begin{array}{l}\text { Poroids } \\
\text { per } 1 \mu \mathrm{m}\end{array}$ & $\begin{array}{l}\text { Poroid } \\
\text { structure }\end{array}$ & $\begin{array}{l}\text { Species } \\
\text { ID }\end{array}$ \\
\hline Phytoplankton (6) & $\begin{array}{l}\text { Linear- } \\
\text { lanceolate }\end{array}$ & $88-93$ & $3.7-4.2$ & Absent & $11-12$ & $11-13$ & 2 & 3 & nd & P. pungens \\
\hline Whale feces (12) & nd & nd & $2.8-4.0^{\mathrm{a}}$ & nd & $10-14$ & $10-14$ & 2 & 3 & nd & P. pungens \\
\hline Phytoplankton (6) & $\begin{array}{l}\text { Lanceolate- } \\
\text { asymmetrical }\end{array}$ & $89-127$ & $5.5-7.0$ & Absent & $17-20$ & $16-19$ & $3-4$ & $7-9$ & Simple & P. seriata \\
\hline Whale feces (3) & nd & nd & nd & nd & 20 & 19 & 3 & $7-9$ & nd & P. seriata \\
\hline Whale feces (17) & nd & nd & $1.1-1.6^{\mathrm{a}, \mathrm{b}}$ & Present & $34-43$ & $20-26$ & 1 & $5-6$ & nd & $\begin{array}{l}\text { P. pseudodelicatissima- } \\
\text { complex }^{\mathrm{e}}\end{array}$ \\
\hline Phytoplankton (6) & Lanceolate & $87-93$ & $4.9-6.8$ & Present & $21-24$ & $20-22$ & $2-3^{c}$ & $5-6$ & Complex & $P$. fraudulenta \\
\hline Whale feces (5) & nd & nd & $4.1-5.1^{\mathrm{a}}$ & nd & $21-24$ & $19-22$ & 2 & $5-6$ & nd & P. fraudulenta \\
\hline Phytoplankton (5) & $\begin{array}{l}\text { Lanceolate- } \\
\text { asymmetrical }\end{array}$ & $39-64$ & $4.9-5.4$ & Present & $29-32$ & $16-18$ & 2 & 9 & nd & P. subpacifica \\
\hline Whale feces (2) & nd & nd & $4.0^{\mathrm{a}}$ & nd & 28 & nd & 2 & 9 & nd & P. subpacifica \\
\hline Phytoplankton (5) & $\begin{array}{l}\text { Linear- } \\
\text { lanceolate }\end{array}$ & $63-73$ & $2.0-3.2$ & Present & $19-28$ & $13-16$ & $2-3^{c}$ & $8-9$ & Simple & P. turgidula ${ }^{\mathrm{d}}$ \\
\hline Whale feces (26) & nd & nd & $1.8-2.5^{\mathrm{a}, \mathrm{b}}$ & Present & $20-29$ & $10-16$ & $2-3^{c}$ & $7-11$ & Simple & P. cf. turgidula \\
\hline Phytoplankton (5) & Lanceolate & $66-74$ & $1.4-1.5$ & Present & $37-38$ & $20-25$ & 2 & $10-11$ & Simple & P. delicatissima \\
\hline Whale feces (6) & nd & nd & $0.8-1.7^{\mathrm{a}, \mathrm{b}}$ & nd & $37-40$ & $20-25$ & 2 & $8-10$ & Simple & P. delicatissima \\
\hline Phytoplankton (8) & Linear & $72-74$ & $1.0-1.5$ & Present & $38-41$ & $21-25$ & 1 & $5-6$ & $4-8$ & P. cf. calliantha \\
\hline Phytoplankton (4) & Lanceolate & $61-67$ & $1.2-1.3$ & Present & $38-41$ & $20-22$ & 1 & $5-6$ & 2 & P. cuspidata \\
\hline Phytoplankton (5) & Linear & 72 & $1.0-1.9$ & Present & $37-40$ & $21-24$ & 1 & 5 & 2 & P. pseudodelicatissima \\
\hline Whale feces (5) & nd & nd & $1.3-1.5$ & nd & $33-34$ & $15-17$ & 1 & $4-5$ & $2-4$ & $\begin{array}{l}\text { Undescribed } \\
\text { species }\end{array}$ \\
\hline Whale feces (1) & nd & nd & $5.1^{\mathrm{a}}$ & nd & 11 & 10 & 4 & 5 & nd & P. multiseries \\
\hline \multicolumn{11}{|c|}{$\begin{array}{l}{ }^{a} \text { Width measured from an unknown section of each cell } \\
{ }^{\mathrm{b}} \text { At least one measurement through central interspace } \\
{ }^{\mathrm{c}} \text { Two rows of poroids most common } \\
\text { dEnlargement present in central portion of cell }\end{array}$} \\
\hline
\end{tabular}

tissima, P. cuspidata, P. calliantha, and P. caciantha (Lundholm et al. 2003), were present in Bay of Fundy, Great South Channel, and Cape Cod Bay fecal samples (Table 4). No TEM data for species within the P. pseudodelicatissima-complex were available from fecal samples. Nonetheless, TEM analyses revealed the presence of P. cuspidata (Fig. 3B) and P. pseudodelicatissima (Fig. 3D) in Bay of Fundy and Roseway Basin phytoplankton samples (ID: P05-128 \& P05-31) collected in August 2005 (Table 3). P. calliantha (Fig. 3A), here reported as $P$. cf. calliantha due to it having fewer hymen poroid divisions (4 to 8 sections) (Table 5) than is typical for this species (Lundholm et al. 2003), was also observed in the Roseway Basin sample (Table 3). $P$. pungens (Fig. 2A) frustule fragments observed in one Bay of Fundy fecal sample collected in September 2005 (ID: 05-057) were also seen in one Georges Bank phytoplankton sample (ID: P05-81) in August of that year (Tables 3 \& 4). TEM micrographs resembling those of an undescribed Pseudo-nitzschia sp. (Fig. 3E) collected in 2004 by Dr. V. Trainer (NOAA Fisheries, Seattle, WA, USA) and colleagues from the northeast USA Pacific coast (N. Lundholm unpubl. obs.) were observed in fecal sample ID number 05-057 (Table 4). $P$. fraudulenta (Fig. 2F and inset) was observed in Bay of Fundy phytoplankton sample ID number P05-128 (August 2005) and in Cape Cod Bay fecal sample ID number 06-001 (April 1999), while P. multiseries (Fig. 2E) was present only in the latter sample (Tables 3 \& 4). The presence of Pseudo-nitzschia spp. was confirmed in several other 2005 Bay of Fundy, Roseway Basin, and Georges Bank phytoplankton samples; however, EM was not performed for confirmation of species identification (Table 3). The predominant Pseudo-nitzschia taxa present in both phytoplankton and whale feces collected in 2005 were members of the P. pseudodelicatissima-complex (Tables 3 \& 4).

Whale feces collected in late August through midSeptember 2006 in the Bay of Fundy contained frustules of Pseudo-nitzschia pungens, P. subpacifica, and $P$. fraudulenta (Table 4; Figs. 2A inset, 2C inset \& 2F inset, respectively). These species were also observed in 2006 Bay of Fundy phytoplankton samples collected from mid-August through early September (Table 3; Figs. 2A inset, C inset \& F inset). Only P. pungens was observed in our single 2006 Roseway Basin fecal sam- 
ple (ID: 06-028) collected in mid-September (Table 4). The most abundant Pseudo-nitzschia spp. in both sample types for 2006 was P. pungens.

Copepod fragments, including appendages, antennae, carapaces, and mouth parts, were present in all $2005(\mathrm{n}=11)$ and $2006(\mathrm{n}=9)$ Eubalaena glacialis fecal samples examined by SEM. Only copepod mouth parts provided sufficient information for species-level identification. Species determinations based on tooth morphology of copepod mandibles were performed
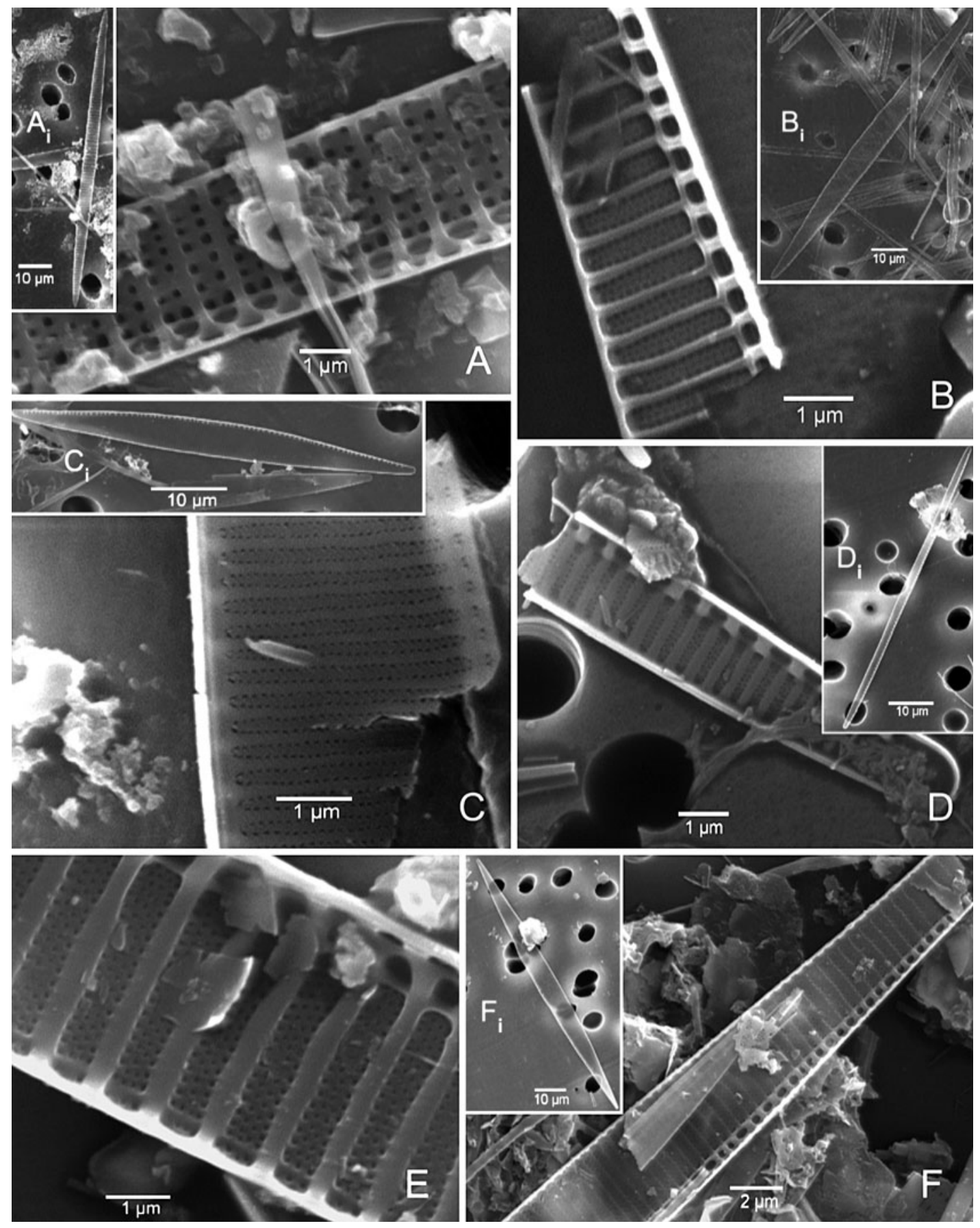

Fig. 2. Pseudo-nitzschia spp. Scanning electron microscope (SEM). Frustule fragments present in Eubalaena glacialis fecal samples (insets: whole valve images of different cells of the same species observed in phytoplankton samples). Species identification:

(A) P. pungens; (B) P. seriata; (C) P. subpacifica; (D) P. cf. turgidula (inset: P. turgidula); (E) P. multiseries; (F) P. fraudulenta 


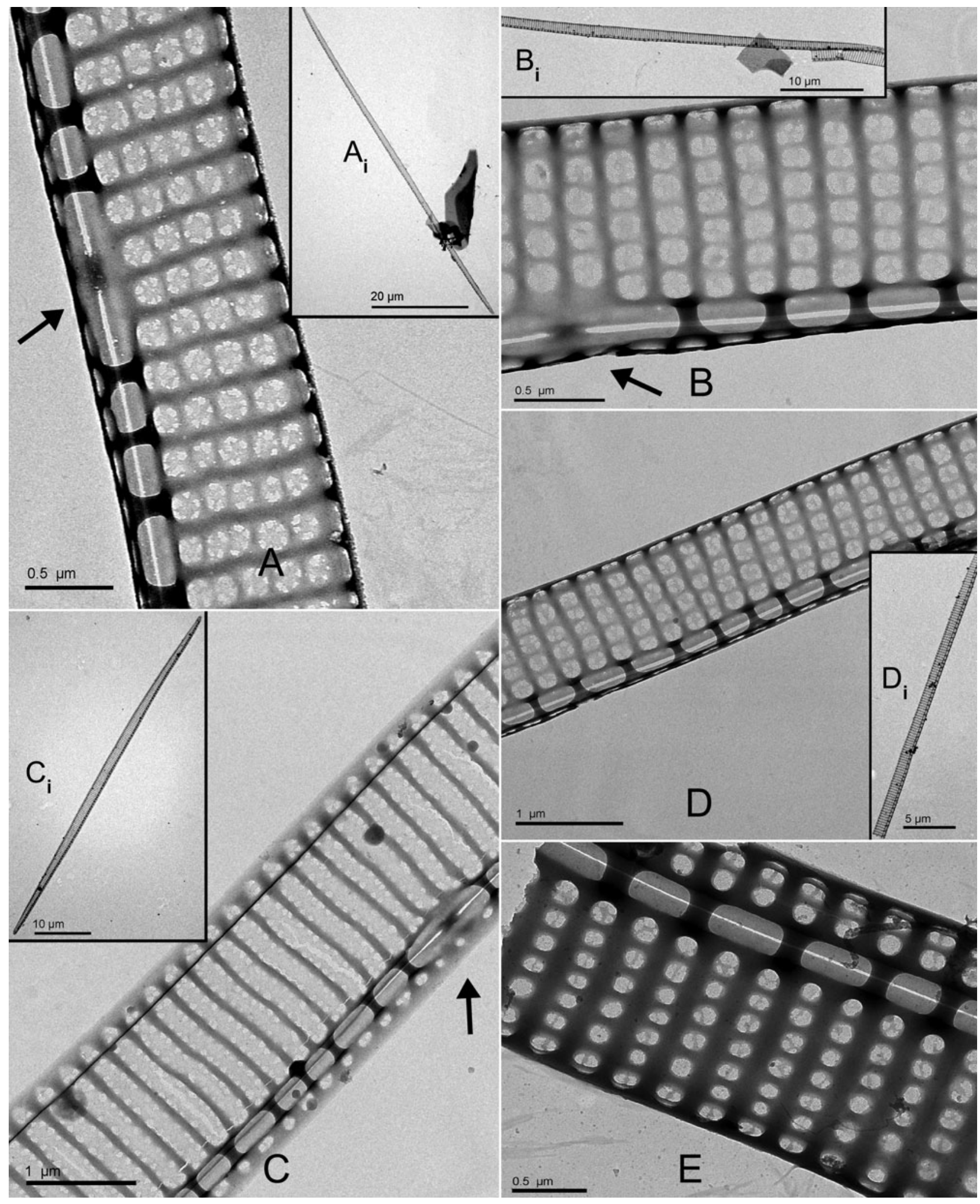

Fig. 3. Pseudo-nitzschia spp., Transmission electron microscope (TEM). Higher (A-C) and lower (inset) magnification micrographs of the same cell present in phytoplankton samples. Arrows point towards the central nodule. (D) Higher and lower (inset) magnification micrographs of different cells of the same species present in phytoplankton samples. (E) Cell fragment observed in Eubalaena glacialis feces. Species identification: (A) P. cf. calliantha; (B) P. cuspidata; (C) P. delicatissima; (D) P. pseudodelicatissima; (E) undescribed Pseudo-nitzschia spp. 


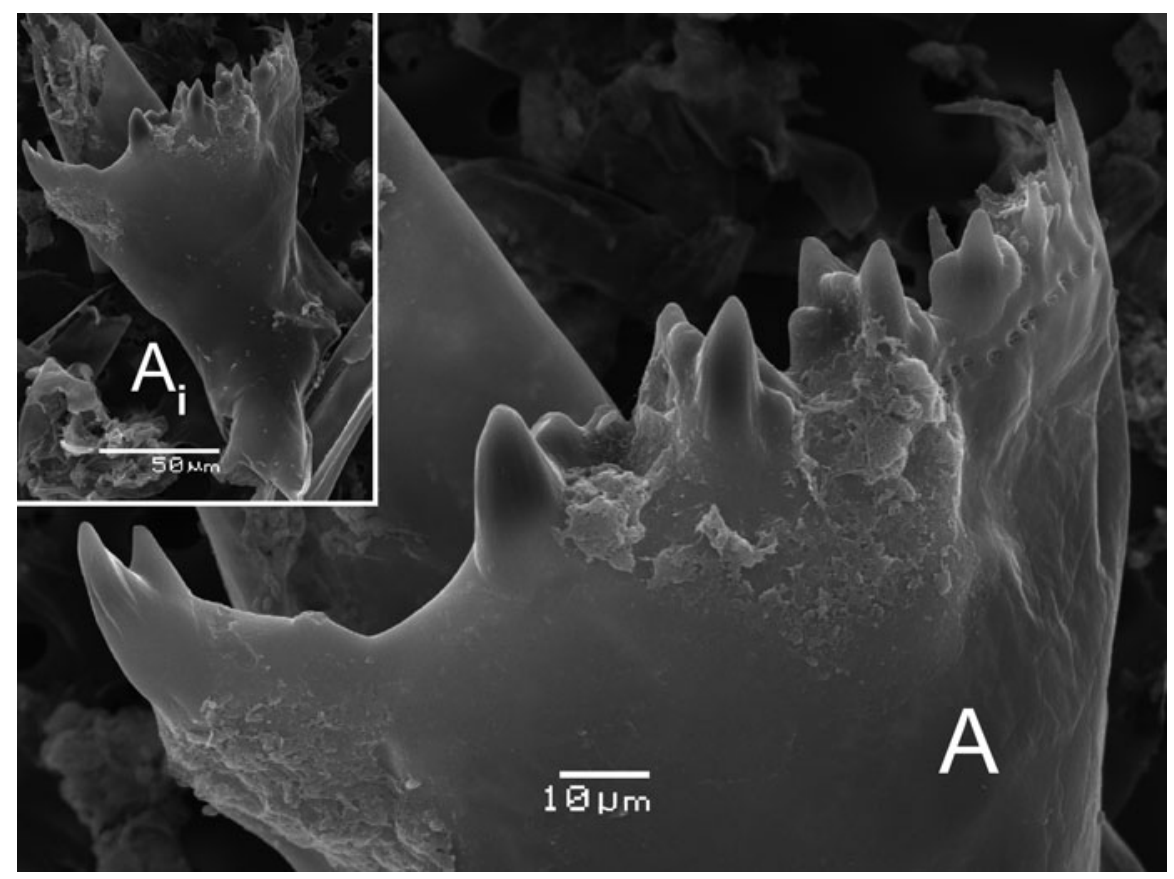

Fig. 4. Calanus finmarchicus. Scanning electron microscope (SEM). Higher (A) and lower (inset) magnification micrographs of a representative copepod mandible present in Eubalaena glacialis fecal samples

according to the criteria of Vyshchkvarceva (1976). Only C. finmarchicus mouth parts (Fig. 4) were identified in all fecal samples analyzed.

\section{DISCUSSION}

\section{DA in right whales}

Our findings demonstrate for the first time that North Atlantic right whales Eubalaena glacialis are exposed to DA by ingesting the copepod vector, Calanus finmarchicus, while on their spring and summer feeding grounds in the Great South Channel and the Bay of Fundy. All E. glacialis fecal samples obtained in 2005 and 2006 ( $\mathrm{n}=70$ ), as well as fecal material collected from 2 stranded individuals (1999 and 2005), tested positive for this neurotoxin by at least one method of analysis, and levels ranged from 0.02 to $0.61 \mathrm{\mu g} \mathrm{DA} \mathrm{g}^{-1}$ (SPR data). These DA levels are at least 300-fold lower than the highest concentrations reported in California sea lion feces (from 1.31 to $182 \mu \mathrm{g} \mathrm{DA} \mathrm{g}^{-1} ; \mathrm{n}=3$ ) during a mass mortality event in 1998 (Scholin et al. 2000). Such disparate DA levels between these 2 studies are not surprising, considering the latter event occurred during an intense Pseudo-nitzschia australis bloom (>100000 cells $\mathrm{l}^{-1}$ ), while total Pseudo-nitzschia spp. cell numbers in the area of right whale feeding grounds were orders of magnitude lower. In addition,
P. australis, a strong DA producer and the Pseudonitzschia species most frequently responsible for ASPrelated problems on a global scale (Bates 2000), was not observed in any of our samples. Lefebvre et al. (2002) also found high DA levels in the feces of 2 blue whales (25 and $207 \mu \mathrm{g} \mathrm{DA} \mathrm{g}{ }^{-1}$ ) and one humpback whale $\left(10 \mu \mathrm{g} \mathrm{DA} \mathrm{g}{ }^{-1}\right)$ collected during a $P$. australis bloom in Monterey Bay, CA, USA. Concentrations of DA in the viscera of anchovies and sardines collected during this $P$. australis bloom ranged from 75 to $444 \mu \mathrm{g}$ DA $\mathrm{g}^{-1}$, greatly exceeding levels measured in our zooplankton samples (from 0.02 to $0.2 \mu \mathrm{g} \mathrm{DA} \mathrm{g}^{-1}$ ) and consistent with the lower DA levels in right whale fecal samples. More recently, DA levels similar to those found in E. glacialis feces were reported in pygmy (Kogia breviceps) and dwarf (Kogia sima) sperm whale feces (median values: $0.078[\mathrm{n}=29]$ and $0.065[\mathrm{n}=12]$ $\mu \mathrm{g}$ DA $\mathrm{g}^{-1}$, respectively) collected from stranded animals along the USA eastern seaboard from 1997 through 2008, although neither the cause of death nor DA source has been established (Fire et al. 2009).

Although the quantity of DA present in North Atlantic right whale fecal samples was generally low, the current findings provide compelling evidence for the potentially chronic exposure of Eubalaena glacialis to this biotoxin for periods of up to 6 mo (April through September). To assess the potential implications of DA exposure to right whale health, estimates of toxin exposure are needed. Durbin et al. (2002) calculated, 
on the basis of in situ feeding rates, that a right whale on average ingests about $4.61 \times 10^{8}$ copepods $\mathrm{d}^{-1}$. Given an average Calanus finmarchicus wet weight of $1.7 \mathrm{mg} \mathrm{copepod}^{-1}$ (Durbin et al. 2002) and the DA levels in our copepod samples, we can expect that right whales ingest between 0.3 and $3.4 \mu \mathrm{g} \mathrm{DA} \mathrm{kg} \mathrm{d}^{-1}(\mathrm{n}=$ 32) for an average $40000 \mathrm{~kg}$ whale (Kenney et al. 1986). This amount is much lower than the quantities of DA ingested by humans suffering from ASP-related symptoms after eating contaminated shellfish (Perl et al. 1990). Our estimates are also orders of magnitude lower than those calculated by Lefebvre et al. (2002) for the humpback (1.1 mg DA kg-1) and blue (0.62 mg DA $\mathrm{kg}^{-1}$ ) whales noted above.

Laboratory studies have shown varying degrees of DA sensitivity in rodents and primates. Levels of 5 to $6 \mathrm{mg}$ DA kg$~^{-1}$ caused neurotoxicity in monkeys, whereas much higher doses $\left(\geq 70 \mathrm{mg} \mathrm{DA} \mathrm{kg}{ }^{-1}\right)$ were required to produce similar symptoms in rats (reviewed in Todd 1993). It has been suggested that marine mammals may be more sensitive to neurotoxin exposure while diving, during which their blood is channeled primarily to vital organs such as the brain and heart, while bypassing major detoxifying organs such as the liver and kidneys (Geraci et al. 1989). This physiological adaptation can thus potentially concentrate a neurotoxin such as DA to elevated levels in the brain where it is most detrimental, posing an obvious concern for right whales that typically perform deep dives over extended periods (i.e. 8 to $16 \mathrm{~min}$ ) to feed (Baumgartner \& Mate 2003). To date, the oral dose of DA required to induce neurotoxic symptoms in right whales or in any other marine mammal remains unknown.

The many DA-associated marine mammal and bird mortality events on the US west coast demonstrate the potential impact of major toxic Pseudo-nitzschia blooms on wildlife populations (Work et al. 1993, Lefebvre et al. 1999, Scholin et al. 2000). Certainly a bloom of similar magnitude occurring on the Eubalaena glacialis summer feeding grounds could be disastrous to this population of approximately 350 to 400 individuals. However, a recent investigation of DA exposure in California sea lions provides a framework for assessing the potential consequences to E. glacialis of being exposed on virtually an annual basis to sublethal doses of this algal biotoxin. A 10-year study of California sea lions stranding at times not associated with acute DA poisoning events revealed that animals exhibited chronic neurological problems (i.e. epilepsy) with neuropathology consistent with DA exposure (Goldstein et al. 2008). In addition, the fact that a subgroup of acutely exposed sea lions developed these chronic neurological symptoms after weeks of remission while held in captivity led these authors to pro- pose that sub-lethal DA poisoning can progress to a chronic disease. It was also argued that the specific nature of hippocampal damage in sea lions exposed chronically to DA may interfere with their navigational abilities. A similar effect of this toxin on North Atlantic right whales, while hypothetical, could enhance their susceptibility to other factors well-documented as primary causes of mortality in this population (e.g. ship collisions, fishing gear entanglement; Reeves et al. 2001).

Exposure to algal biotoxins such as DA is among the proposed contributors to reproductive challenges currently faced by Eubalaena glacialis (Kraus et al. 2007) and is of particular interest given the recently reported link between California sea lion exposure to DA and reproductive failure (Brodie et al. 2006). These animals experienced much higher toxin levels than those estimated herein for E. glacialis. However, DA is actually cleared rapidly in adults but not cleared effectively from the fetal unit (Maucher \& Ramsdell 2007), and the fetus is thus considered to be more susceptible to DA poisoning on the basis of exposure during pregnancy. In fact, fetal poisoning was proposed by Ramsdell \& Zabka (2008) to have a more prominent effect later in life, manifest as a greater likelihood of developing neurological disease. Exposure of E. glacialis females to DA during the several months spent feeding in the Bay of Fundy and adjacent waters can coincide with various stages of pregnancy (approx. 6 to 9 mo), based on a 12 to 13 mo gestation period and a calving period lasting from early December through the end of March in waters off the southeast USA (see Kraus et al. 2007). The potential for fetal DA poisoning and its associated effects should therefore be a consideration in evaluating the possible impacts of algal biotoxins on this endangered N. Atlantic right whale population. Given the limitations inherent in sampling and studying endangered marine mammals, attempts to model the exposure of these whales to DA as well as predict its toxicological effects are needed to assess the implications of long-term, sub-lethal DA exposures for $E$. glacialis health and reproduction.

\section{DA in zooplankton}

The detection of DA in all copepod samples $(\mathrm{n}=32)$ by SPR was an unexpected, yet significant finding of this study. It should be noted that field populations of Calanus finmarchicus used recently in grazing experiments (Leandro et al. 2009) were tested by SPR and found to be negative for DA, indicating that matrix effects were likely not responsible for the uniformly positive responses obtained herein. As in the related 
work of Durbin et al. (2002) and Doucette et al. (2006), all copepod samples examined for species composition (from 76 to $93 \% ; n=9$ ) were dominated largely by Stage V copepodites of C. finmarchicus (Swaim et al. 2009). These results, together with the ubiquitous presence of intact $C$. finmarchicus mandibles in right whale fecal samples, provide convincing evidence for the transfer of DA from Pseudo-nitzschia spp. to E. glacialis via the whales' primary copepod prey. Nonetheless, Pseudo-nitzschia spp. often form long chains several hundred micrometers in length, easily exceeding the average right whale baleen mesh size (335 $\mu \mathrm{m}$; Mayo et al. 2001). Thus, we cannot exclude the possibility that E. glacialis may also be exposed directly to DA by ingesting chains of toxic Pseudonitzschia spp. However, we would argue that the presence of only Pseudo-nitzschia cell fragments, as opposed to whole frustules, in all fecal material examined indicates that the predominant route for DA transfer to E. glacialis is via their copepod prey. C. finmarchicus does not ingest whole diatom cells while feeding, but instead chews and breaks them into smaller pieces using labral teeth and mandibles (Marshall \& Orr 1972). Moreover, grazing experiments conducted recently by Leandro et al. (2009) demonstrated that $C$. finmarchicus is capable of ingesting toxic Pseudo-nitzschia cells and does not discriminate between toxic and non-toxic species when both are available. The Pseudo-nitzschia cell fragments present in right whale feces are thus more likely derived from copepod gut contents. Similar results were reported by Lefebvre et al. (2002), which showed the presence of $P$. australis cell fragments in blue whale feces and led the authors to suggest that this material was likely derived from krill gut contents. In contrast, micrographs showing whole $P$. australis frustules in California sea lion feces after the sea lions had fed on DA-contaminated anchovies were consistent with the fact that these planktivorous fish, unlike copepods or krill, ingest whole Pseudo-nitzschia cells by means of a 'feeding basket' or sieve (Lefebvre et al. 1999). In addition to the ubiquitous presence of DA in copepod material, all krill samples analyzed $(n=6)$ also contained this neurotoxin, which agrees with previous studies confirming the ability of krill to act as vectors for DA transfer in marine food webs (Bargu et al. 2002, Lefebvre et al. 2002, Bargu \& Silver 2003). Bargu et al. (2002) reported concentrations as high as $44 \mu \mathrm{g}$ DA eq $\mathrm{g}^{-1}$ tissue in krill collected during the 2000 P. australis bloom in Monterey Bay, CA. While DA levels measured in zooplankton during the present study were much lower than those found by Bargu et al. (2002), values for the former would undoubtedly have been much higher in the presence of a toxic Pseudo-nitzschia bloom.

\section{Methods of DA analysis}

A comparison of the 3 methods used to measure DA in zooplankton and fecal samples revealed that while SPR and RBA estimates were comparable to each other, LC-MS/MS values where generally much lower (Tables 1 \& 2). While this discrepancy cannot be explained fully at this time, further LC-MS/MS analyses have revealed the presence of a putative methylated DA metabolite, in both whale feces and zooplankton extracts (Wang et al. unpubl. data, Leandro et al. 2009). Thus far, there is no evidence to suggest that this compound is produced directly by Pseudonitzschia spp., which supports the idea that this compound is formed via metabolism of the toxin. The presence of this methylated DA metabolite in all zooplankton as well as all right whale fecal samples tested to date provides further evidence that copepods are the proximate source of DA for right whales; however, the possibility that this compound may be formed de novo in the right whale digestive system cannot be discounted. The DA LC-MS/MS concentrations reported in this study represent only those of the parent DA molecule and do not take into account the DA metabolite present in these samples, which has been estimated to represent as much as $50 \%$ and $30 \%$ of total DA in fecal and zooplankton samples, respectively (assuming that DA and its methylated metabolite have similar ionization and fragmentation efficiency). If this metabolite is determined to react equivalently to DA with both the SPR antibody and RBA glutamate receptors (yet to be confirmed), this could explain why DA measurements by the latter 2 methods were generally higher. Implications of the metabolite's presence in right whales, copepods, or krill await determination of its toxicity relative to the parent toxin.

\section{Pseudo-nitzschia spp. in the Bay of Fundy}

The present study provides important insights into the occurrence of several potentially toxic Pseudonitzschia species in both phytoplankton and right whale feces originating from the Bay of Fundy and nearby locations during 2005 and 2006. P. pungens, $P$. delicatissima, $P$. turgidula $(P$. cf. turgidula in whale feces), $P$. fraudulenta, $P$. subpacifica, and $P$. seriata were present in both sample types, while $P$. cf. calliantha, P. cuspidata, and P. pseudodelicatissima could be verified only in phytoplankton samples. Of the 9 mentioned Pseudo-nitzschia species, only P. seriata (Lundholm et al. 1994, Bates et al. 2002, Fehling et al. 2004), and P. cf. calliantha (Martin et al. 1990, Bates et al. 1998), identified previously by these authors as $P$. pseudodelicatissima (Lundholm et al. 2003), have 
been shown to both produce DA in culture and be associated with shellfish closures in N. Atlantic waters. P. seriata, initially reported as non-toxic along the east coast of Canada (Bates et al. 1989), has recently been shown to produce DA in western European waters (Lundholm et al. 1994, Fehling et al. 2004). P. seriata was also responsible for high DA levels in molluscs from the St. Lawrence region of Canada between 1998 and 2000 (Couture et al. 2001) and for a shellfish harvesting closure in Prince Edward Island, Canada, during 2002 (Bates et al. 2002). Interestingly, this species, which was not observed in the Bay of Fundy from 1998 through 2001 (Kaczmarska et al. 2005), was present in several Bay of Fundy and Great South Channel samples (phytoplankton and feces) collected in 2005.

Other potential DA producers identified from the 2005 samples were Pseudo-nitzschia delicatissima, P. cuspidata and P. pseudodelicatissima. Some, but not all, cultures of these 3 species have been shown to produce DA (reviewed in Bates et al. 1998, Lundholm et al. 2003, Bill et al. 2005), yet none has been associated with DA intoxication events or shellfish harvesting closures. P. turgidula, also observed in the 2005 phytoplankton samples, was reported previously to synthesize small amounts of DA in culture (Rhodes et al. 1996); however, the identity of this isolate was later questioned (Rhodes et al. 1998). The distribution of $P$. turgidula in the North Atlantic is poorly described (Hasle 2002), and the present study is the first to confirm identification of the taxon in the phytoplankton from this region. The fragments of $P$. turgidula-like frustules (no whole cells) present in whale feces exhibited lower striae and fibulae counts than the morphologically similar $P$. delicatissima but were sufficiently similar to $P$. turgidula for classification as $P$. cf. turgidula. Further work is needed to determine the ability of $P$. turgidula to produce DA in both culture and natural populations. Interestingly, fragments resembling those of an undescribed Pseudo-nitzschia species observed by V. Trainer and colleagues off the northeast Pacific US coast (N. Lundholm unpubl. data) occurred in one right whale fecal sample, although its ability to produce DA remains uncertain. Also notable was the observation that only fecal material from the whale stranding in 1999 contained the typically strong DA producer $P$. multiseries, yet exhibited among the lowest toxin values herein (Table 1). However, this is consistent with the fact that only a single $P$. multiseries fragment along with remnants of 2 other weakly toxic species (i.e. P. pseudodelicatissima-complex, $P$. fraudulenta) were documented in this fecal sample (Tables $4 \& 5$ ).

Pseudo-nitzschia pungens, $P$. fraudulenta, and $P$. subpacifica were present in the 2006 phytoplankton and fecal samples, and all 3 taxa have been reported previously from the Bay of Fundy (Kaczmarska et al. 2005). While none of these species is a typical DA producer in the North Atlantic (Bates 2000, Bates \& Trainer 2006), all Bay of Fundy and Roseway Basin fecal $(n=24)$ and zooplankton $(\mathrm{n}=27)$ samples collected in 2006 contained this neurotoxin. Although cultures of $P$. pungens originating from New Zealand (Rhodes et al. 1996), Washington state (Trainer et al. 1998), and Monterey Bay (Bates et al. 1998) have occasionally produced small amounts of DA, this species has consistently been reported as non-toxic in the Atlantic Ocean, Gulf of Mexico, and European waters (reviewed by Bates 2000). P. fraudulenta is a less studied taxon; nonetheless, Rhodes et al. (1998) demonstrated low DA production by a New Zealand isolate (0.02 pgDA cell ${ }^{-1}$ ) confirmed as this taxon by both sequencing and electron microscopy. There have thus far been no reports of a toxigenic $P$. subpacifica isolate (Bates \& Trainer 2006). Given the detection of DA in all 2006 zooplankton and fecal samples, coupled with the occurrence of $P$. pungens and/or $P$. fraudulenta in all 2006 fecal and phytoplankton samples, it is likely that at least one of these Pseudo-nitzschia species represents the toxin source. The inability to detect DA in any of these phytoplankton samples likely reflects patchy distributions and low cell concentrations at the time/location of sampling, which never exceeded $\sim 10000$ cells $\mathrm{l}^{-1}$ and are far below that typical of Pseudo-nitzschia bloom conditions ( $\geq 100000$ cells $1^{-1}$; Bates et al. 1998). For both 2005 and 2006, the possibility remains that toxigenic Pseudo-nitzschia spp. not observed in the limited number of samples examined during this study contributed to DA levels measured.

\section{CONCLUSIONS}

The present study is the first to demonstrate that the endangered North Atlantic right whales Eubalaena glacialis are exposed to the neurotoxin domoic acid through the ingestion of contaminated copepods while in their spring and summer feeding areas. While both the toxin concentrations measured in feces and estimated DA ingestion levels are lower than those published for other marine mammals, our data establish that right whales were potentially exposed to DA for periods up to 6 mo annually. The detection of DA in all copepod samples (largely dominated by $C$. finmarchicus) and the presence of $C$. finmarchicus mandibles as well as fragments (no whole cells) of Pseudo-nitzschia spp. in right whale feces clearly point towards this copepod as a primary vector for DA transfer into right whales. Several potentially toxic Pseudo-nitzschia spp. were collected close 
to feeding whales and represent the most likely source of DA.

Future efforts should focus on the development of appropriate animal models for characterizing the potential health effects of DA toxicity on Eubalaena glacialis, since direct experimentation with right whales is not feasible. Development of exposure and risk assessment models will also aid in projecting the susceptibility of E. glacialis to DA and other algal biotoxins, such as PSP. Finally, additional work is required to determine the relative toxicity of the methylated DA metabolite, present in our zooplankton and fecal samples, with respect to the parent toxin.

Acknowledgements. We thank K. King for assistance with DA extraction from fecal materials and Dr. S. Morton for training L. Leandro to conduct SEM analyses. We acknowledge members of the New England Aquarium, L. Murison, Dr. M. Moore, and Dr. M. Baumgartner for assistance with fecal sample collection; Dr. H. Koopman, Dr. A. Westgate, and Z. Swaim for providing zooplankton samples and copepod species abundance data; Dr. T. Cole and B. Keafer for zooplankton and phytoplankton collections; and Dr. D. DeBiase (Univ. di Roma La Sapienza, Italy) for providing the GAD enzyme used in the receptor binding assays. The DA antibody was a gift of Dr. C. Elliott, Queen's University Belfast. We are also grateful to Drs. G. Teegarden and R. Gerber for assistance with copepod species identification and Dr. L. Kracker for GIS map design. We also thank Dr. J. Ramsdell for insightful discussions and critical review of this manuscript. Samples from stranded whales were collected under NE US NMFS MMPA Regional Letters of Authorization, and under NMFS permit to Dr. T. Rowles (no. 932-1489-07). Fecal samples were collected in US waters under NMFS permit to Dr. S. Kraus (655-1652$00)$, and in Canadian waters under Species at Risk Act Scientific Research Permit no. MAR-SA-2005-007 and MAR-SA2006-007. Funding was provided in part by NOAA/NOS operational funds and by an NOAA/NMFS grant (\#EA133F02-SE-0155) to R.M.R. for collection and analysis of right whale fecal material.

Disclaimer: The National Ocean Service (NOS) does not approve, recommend, or endorse any proprietary product or material mentioned in this publication. No reference shall be made to NOS, or to this publication furnished by NOS in any advertising or sales promotion which would indicate or imply that NOS approves, recommends, or endorses any proprietary product or proprietary material mentioned herein or which has as its purpose any intent to cause directly or indirectly the advertised product to be used or purchased because of NOS publication.

\section{LITERATURE CITED}

Bargu S, Silver MW (2003) Field evidence of krill grazing on the toxic diatom genus Pseudo-nitzschia in Monterey Bay, California. Bull Mar Sci 72:629-638

Bargu S, Powell CL, Coale SL, Busman M, Doucette GJ, Silver MW (2002) Krill: a potential vector for domoic acid in marine food webs. Mar Ecol Prog Ser 237:209-216

Bates SS (2000) Domoic-acid-producing diatoms: another genus added! J Phycol 36:978-985
Bates SS, Trainer VL (2006) The ecology of harmful diatoms. In: Granéli E, Turner JT (eds) Ecology of harmful algae, Vol 189. Springer-Verlag, New York, p 81-93

Bates SS, Bird CJ, Freitas ASW, Foxall R and others (1989) Pennate diatom Nitzschia pungens as the primary source of domoic acid, a toxin in shellfish from eastern Prince Edward Island, Canada. Can J Fish Aquat Sci 46: 1203-1215

Bates SS, Garrison DL, Horner RA (1998) Bloom dynamics and physiology of domoic-acid-producing Pseudonitzschia species. In: Anderson DM, Cembella AD, Hallegraeff GM (eds) Physiological ecology of harmful algal blooms. Springer-Verlag, Heidelberg, p 267-292

Bates SS, Léger C, White JM, MacNair N and others (2002) Domoic acid production by the diatom Pseudo-nitzschia seriata causes spring closures of shellfish harvesting for the first time in the Gulf of St. Lawrence, Eastern Canada. Book of abstracts, 10th International Conference on Harmful Algae. ISSHA (International Society for the Study of Harmful Algae), p 23

Baumgartner MF, Mate BR (2003) Summertime foraging ecology of North Atlantic right whales. Mar Ecol Prog Ser 264: 123-135

Baumgartner MF, Mate BR (2005) Summer and fall habitat of the North Atlantic right whales (Eubalaena glacialis) inferred from satellite telemetry. Can J Fish Aquat Sci 62: $527-543$

Baumgartner MF, Cole TVN, Campbell J, Teegarden G, Durbin EG (2003a) Associations between North Atlantic right whales and their prey, Calanus finmarchicus, over diel and tidal time scales. Mar Ecol Prog Ser 264:155-166

> Baumgartner MF, Cole TVN, Clapham PJ, Mate BR (2003b) North Atlantic right whale habitat in the lower Bay of Fundy and on the SW Scotian Shelf during 1999-2001. Mar Ecol Prog Ser 264:137-154

> Berman FW, LePage KT, Murray TF (2002) Domoic acid neurotoxicity in cultured cerebellar granule neurons is controlled preferentially by NMDA receptor $\mathrm{Ca}^{(2+)}$ influx pathway. Brain Res 924:20-29

Bill BD, Lundholm N, Connell L A BK, Trainer VL (2005) Domoic acid in Pseudo-nitzschia cuspidata from Washington State coastal waters. 3rd Symposium on Harmful Algae in the US. MBARI (Monterey Bay Aquarium Research Institute), Moss Landing, CA, p 77

> Brodie EC, Gulland F, Greig DJ, Hunter M and others (2006) Domoic acid causes reproductive failure in California sea lions (Zalophus californianus). Mar Mamm Sci 22:700-707

> Caswell H, Fujiwara M, Brault S (1999) Declining survival probability threatens the North Atlantic right whale. Proc Natl Acad Sci USA 96:3308-3313

Clapham PJ, Young SB, Brownell RL (1999) Baleen whales: conservation issues and the status of the most endangered populations. Mammal Rev 29:37-60

Couture JV, Levasseur M, Bonneau E, Desjardins C and others (2001) Spatial and temporal variation of domoic acid in molluscs and of Pseudo-nitzschia spp. blooms in the St. Lawrence from 1998 to 2000. Can Tech Rep Fish Aquat Sci 2375:24

> Doucette GJ, Cembella AD, Martin JL, Michaud J, Cole TVN, Rolland RM (2006) Paralytic shellfish poisoning (PSP) toxins in North Atlantic right whales Eubalaena glacialis and their zooplankton prey in the Bay of Fundy, Canada. Mar Ecol Prog Ser 306:303-313

> Durbin E, Teegarden G, Campbell R, Cembella A, Baumgartner MF, Mate BR (2002) North Atlantic right whales, Eubalaena glacialis, exposed to paralytic shellfish poisoning (PSP) toxins via a zooplankton vector, Calanus fin- 
marchicus. Harmful Algae 1:243-251

Fehling J, Green DH, Davidson K, Bolch CJ, Bates SS (2004) Domoic acid production by Pseudo-nitzschia seriata (Bacillariophyceae) in Scottish waters. J Phycol 40: $622-630$

Fire SE, Wang Z, Leighfield TA, Morton SL and others (2009) Domoic acid exposure in pygmy and dwarf sperm whales (Kogia spp.) from southeastern and mid-Atlantic US waters. Harmful Algae 8:658-664

Fujiwara M, Caswell H (2001) Demography of the endangered North Atlantic right whale. Nature 414:537-541

> Geraci JR, Anderson DM, Timperi RJ, Aubin DJS, Early GA, Prescott JH, Mayo CA (1989) Humpback whales (Megaptera novaeangliae) fatally poisoned by dinoflagellate toxin. Can J Fish Aquat Sci 46:1895-1898

Goldstein T, Mazet JAK, Zabka TS, Langlois G and others (2008) Novel symptomatology and changing epidemiology of domoic acid toxicosis in California sea lions (Zalophus californianus): an increasing risk to marine mammal health. Proc R Soc Lond B Biol Sci 275:267-276

Greene CH, Pershing AJ (2004) Climate and the conservation biology of North Atlantic right whales: the right whale at the wrong time? Front Ecol Environ 2:29-34

Hamilton PK, Knowlton AR, Marx MK (2007) Right whales tell their own stories: the photo-identification Catalog. In: Kraus SD, Rolland RM (eds) The urban whale: North Atlantic right whales at the crossroads. Harvard University Press, Cambridge, MA, p 75-104

Hasle GR (2002) Are most of the domoic acid-producing species of the diatom genus Pseudo-nitzschia cosmopolites? Harmful Algae 1:137-146

Kaczmarska I, LeGresley MM, Martin JL, Ehrman J (2005) Diversity of the diatom genus Pseudo-nitzschia Pergallo in the Quoddy region of the Bay of Fundy, Canada. Harmful Algae 4:1-19

Kenney RD, Hyman MAM, Owen RE, Scott GP, Winn HE (1986) Estimation of prey densities required by western north Atlantic right whales. Mar Mamm Sci 2:1-13

Kenney RD, Mayo CA, Winn HE (2001) Migration and foraging strategies at varying special time scales in western North Atlantic right whales: a review of hypotheses. J Cetacean Res Manag 2(special issue):251-260

Knowlton AR, Kraus SD (2001) Mortality and serious injury of northern right whales (Eubalaena glacialis) in the western North Atlantic Ocean. J Cetacean Res Manag 2(special issue):193-208

Kraus SD, Rolland RM (2007) Right whales in the urban ocean. In: Kraus SD, Rolland RM (eds) The urban whale: North Atlantic right whales at the crossroads. Harvard University Press, Cambridge, MA, p 1--38

Kraus SD, Hamilton PK, Kenney RD, Knowlton AR, Slay CK (2001) Reproductive parameters of the North Atlantic right whale. J Cetacean Res Manag 2(special issue):321-336

Kraus SD, Pace RM III, Frasier TR (2007) High investment, low return: the strange case of reproduction in Eubalaena glacialis. In: Kraus SD, Rolland RM (eds) The urban whale: North Atlantic right whales at the crossroads. Harvard University Press, Cambridge, MA, p 172-199

Leandro LF, Teegarden GJ, Roth PB, Wang Z, Doucette GJ (2009) The copepod Calanus finmarchicus: a potential vector for trophic transfer of the marine algal biotoxin, domoic acid. J Exp Mar Biol Ecol doi:10.1016/j.jembe. 2009.11.002

Lefebvre KA, Powell CL, Busman M, Doucette GJ and others (1999) Detection of domoic acid in northern anchovies and California sea lions associated with an unusual mortality event. Nat Toxins 7:85-92
Lefebvre KA, Bargu S, Kieckhefer T, Silver MW (2002) From sanddabs to blue whales: the pervasiveness of domoic acid. Toxicon 40:971-977

Lundholm N, Skov J, Pocklington R, Moestrup Ø (1994) Domoic acid, the toxic amino acid responsible for amnesic shellfish poisoning, now in Pseudonitzschia seriata (Bacillariophyceae) in Europe. Phycologia 33:475-478

Lundholm N, Moestrup Ø, Hasle GR, Hoef-Emden K (2003) A study of the Pseudo-nitzschia pseudodelicatissima/cuspidata complex (Bacillariophyceae): What is P. pseudodelicatissima? J Phycol 39:797-813

Lundholm N, Moestrup Ø, Kotaki Y, Hoef-Emden K, Scholin C, Miller P (2006) Inter- and intraspecific variation of the Pseudo-nitzschia delicatissima complex (Bacillariophyceae) illustrated by rRNA probes, morphological data and phylogenetic analyses. J Phycol 42:464-481

Marshall SM, Orr AP (1972) The biology of a marine copepod. Springer-Verlag, New York

Martin JL, Haya K, Burridge LE, Wildish DJ (1990) Nitzschia pseudodelicatissima - a source of domoic acid in the Bay of Fundy, eastern Canada. Mar Ecol Prog Ser 67:177-182

Maucher JM, Ramsdell JS (2007) Maternal-fetal transfer of domoic acid in rats at two gestational time points. Environ Health Perspect 115:1743-1746

Mayo CA, Letcher BH, Scott S (2001) Zooplankton filtering efficiency of the baleen of a North Atlantic right whale, Eubalaena glacialis. J Cetacean Res Manag 2(special issue): $225-229$

> Murison LD, Gaskin DE (1989) The distribution of right whales and zooplankton in the Bay of Fundy, Canada. Can J Zool 67:1411-1420

> Perl TM, Bedard L, Kosatsky T, Hockin JC, Todd EC, Remis RS (1990) An outbreak of toxic encephalopathy caused by eating mussels contaminated with domoic acid. N Engl J Med 322:1775-1780

Ramsdell JS (2007) The molecular and integrative basis to domoic acid toxicity. In: Botana L (ed) Phycotoxins: chemistry and biochemistry. Blackwell Publishing Professional, Cambridge, MA, p 223-250

Ramsdell JS, Zabka TS (2008) In utero domoic acid toxicity: a fetal basis to adult disease in the California sea lion (Zalophus californianus). Mar Drugs 6:262-290

Reeves RR, Rolland RM, Clapham PJ (eds) (2001) Causes of reproductive failure in North Atlantic right whales: new avenues of research. Report of a workshop held 26-28 April, 2000, Falmouth, Massachusetts. Northeast Fisheries Science Center Reference Document 01-16, Woods Hole, MA, p 46

Rhodes L, White D, Syhre M, Atkinson M (1996) Pseudonitzschia species isolated from New Zealand coastal waters: domoic acid production in vitro and links with shellfish toxicity. In: Yasumoto $\mathrm{T}$, Oshima Y, Fukuyo Y (eds) Harmful and toxic algal blooms. Intergovernmental Oceanographic Commission of UNESCO, Paris, p 155-158

Rhodes L, Scholin C, Garthwaite I, Haywood A, Thomas A (1998) Domoic acid producing Pseudo-nitzschia species educed by whole cell DNA probe-based and immunochemical assays. In: Reguera B, Blanco J, Fernández ML, Wyatt $\mathrm{T}$ (eds) Harmful algae. Xunta de Galicia and the Intergovernmental Oceanographic Commission, UNESCO, Paris, p 274-277

Rolland RM, Hamilton PK, Kraus SD, Davenport B, Gillett RM, Wasser SK (2006) Faecal sampling using detection dogs to study reproduction and health in North Atlantic right whales (Eubalaena glacialis). J Cetacean Res Manag 8:121-125

Scholin CA, Gulland F, Doucette GJ, Benson S and others 
(2000) Mortality of sea lions along the central California coast linked to a toxic diatom bloom. Nature 403:80-84

Skov J, Lundholm N, Moestrup Ø, Larsen J (1999) Potentially toxic phytoplankton. 4. The diatom genus Pseudo-nitzschia (Diatomophyceae/Bacillariophyceae). ICES identification leaflets for plankton, Vol 185. ICES, Copenhagen

Swaim ZT, Westgate AJ, Koopman HN, Rolland RM, Kraus SD (2009) Metabolism of ingested lipids by North Atlantic right whales. Endang Species Res 6:259-271

Todd ECD (1993) Domoic acid and amnesic shellfish poisoning - a review. J Food Prot 56:69-83

Trainer VL, Wekell JC, Horner RA, Hatfield C, Stein JE (1998) Domoic acid production by Pseudo-nitzschia pungens. In: Reguera B, Blanco J, Fernández ML, Wyatt T (eds) Harmful algae. Xunta de Galicia and the Intergovernmental Oceanographic Commission, UNESCO, Paris, p 337-340

Traynor IM, Plumpton L, Fodey TL, Higgins C, Elliott CT (2006) Immunobiosensor detection of domoic acid as a screening test in bivalve molluscs: comparison with liquid chromatography-based analysis. J AOAC Int 89:868-872

Van Dolah FM (2005) Effects of harmful algal blooms. In:

Editorial responsibility: John Piatt, Anchorage, Alaska, USA
Reynolds JE III, Perrin WF, Reeves RR, Montgonery S, Ragen TJ (eds) Marine mammal research. John Hopkins University Press, Baltimore, MD, p 85-214

Van Dolah FM, Leighfield TA, Haynes BL, Hampson DR, Ramsdell JS (1997) A microplate receptor assay for the amnesic shellfish poisoning, domoic acid, utilizing a cloned glutamate receptor. Anal Biochem 245:102-105

Vyshchkvarceva NV (1976) The functional morphology of mouth parts of the species Calanus s.l. (Copepoda, Calanoida). In: Brodskij KA, Vyshchkvarceva NV (eds) Functional morphology, growth and development of invertebrates of sea plankton. Nauka, Leningrad, p 11-69 (in Russian)

Wang Z, King KL, Ramsdell JS, Doucette GJ (2007) Determination of domoic acid in seawater and phytoplankton by liquid chromatography-tandem mass spectrometry. J Chromatogr A 1163:169-176

Work TM, Barr B, Beale AM, Fritz L, Quilliam MA, Wright JLC (1993) Epidemiology of domoic acid poisoning in brown pelicans (Pelecanus occidentalis) and Brandt's cormorants (Phalacrocorax penicillatus) in California. J Zoo Wildl Med 24:54-62

Submitted: March 12, 2009; Accepted: September 15, 2009 Proofs received from author(s): November 25, 2009 